\title{
Costs, Benefits, Parasites and Mutualists: The Use and Abuse of the Mutualism-Parasitism Continuum Concept for Epichloë Fungi
}

\author{
Jonathan A. Newman, ${ }^{* \dagger}$ Sierra Gillis ${ }^{* \ddagger}$, and Heather A. Hager*§
}

\begin{abstract}
The species comprising the fungal endophyte genus Epichloë are symbionts of cool season grasses. About half the species in this genus are strictly vertically transmitted, and evolutionary theory suggests that these species must be mutualists. Nevertheless, Faeth and Sullivan (e.g., 2003) have argued that such vertically transmitted endophytes are "usually parasitic," and Müller and Krauss (2005) have argued that such vertically transmitted endophytes fall along a mutualism-parasitism continuum. These papers (and others) have caused confusion in the field. We used a systematic review to show that close to half of the published papers in this field incorrectly categorize the interaction. Here, we develop the argument that advantages and disadvantages are not the same things as mutualism and parasitism and that experimental evidence must be interpreted in the context of theory. We argue that, contra Faeth and Sullivan, it is highly unlikely that such strictly vertically transmitted endophytes can be parasites, and that, contra Müller and Krauss, it is inappropriate to apply the continuum concept to strictly vertically transmitted endophytes. We develop a mathematical model to support and illustrate our arguments. We use the model to clarify that parasitism requires some degree of horizontal transmission, and that while it is appropriate to use the continuum concept when referring to the genus as a whole, or to species that possess horizontal transmission, we argue that it is inappropriate to use the concept when referring to species that are strictly vertically transmitted.
\end{abstract}

\section{Keywords}

mutualism $\bullet$ endophytes, $\bullet$ Epichloë $・$ Neotyphodium $\bullet$ vertical transmission

${ }^{*}$ Department of Biology, Wilfrid Laurier University, 75 University Avenue West,

Waterloo, Ontario, Canada, N2L 3C2; $\dagger$ jonathannewman@wlu.ca (iD https://orcid.org/0000-0003-3155-4084;

¥ iDhttps://orcid.org/0000-0002-3418-4958; § (iD https://orcid.org/0000-0002-0066-6844

Received 29 July, 2020; Accepted 23 June, 2021

doi:10.3998/ptpbio.2103 


\section{Introduction}

In this paper, we are concerned with the nature of the ecological interaction between fungal species in the genus Epichloë and their grass host plants. In particular, we consider the existence of a 'mutualism-parasitism continuum' for these species. We begin by defining some terms. Within the context of pairwise species interactions, there are 'consumer interactions' in which one interacting species benefits and the other is harmed $(+,-)$. These are commonly termed predation, parasitism, herbivory, etc. In commensalism, one species benefits and the other is not affected $(+, 0)$. In ammensalism, one species is harmed and the other is not affected $(-, 0)$. And finally, mutualism occurs when both species benefit from the interaction $(+,+)$. We distinguish the use of these terms from fitness costs and benefits. The costs and benefits of a particular species interaction may shift with species ontogeny, environment, or climate. There may be times during the lifespan of an individual where the costs outweigh the benefits, and other times where the benefits outweigh the costs. As Ewald (1987, 295) succinctly put it:

The net effect on the host's inclusive fitness of all harmful and beneficial characteristics is the theoretical criterion used to assign the labels parasitism, commensalism, or mutualism.

That is, it is the integration of the fitness benefits minus the costs, over the lifespan of the individual, that determines the signs used above to describe the pairwise interaction. Furthermore, we stipulate that in order to conclusively, empirically demonstrate the nature of such a pairwise interaction, one would need to measure and compare individual fitness over the lifetime of the individuals involved. Short-term experiments that measure an aspect of, or a surrogate of, fitness provide some information about the immediate costs and benefits of the relationship, but such experiments do not demonstrate the underlying nature of the interaction. Since individual experiments rarely actually measure lifetime fitness, conclusions as to the nature of the interaction are most often inferences based on empirical demonstrations of costs and benefits coupled with evolutionary and ecological theory.

\subsection{Epichloë Fungi}

The grass subfamily Poöideae arose 30-40 million years ago, in conjunction with a monophyletic clade of systemic endophytic fungi in the genus Epichloë. These fungi colonize most aboveground grass tissues, but are usually absent from root tissues. Some Epichloë species cause 'choke disease' whereby the fungus suppresses the host's seed production, using the culms as a site to produce fungal ascospores that disperse to infect new host plants. However, many Epichloë are also able to asymptomatically colonize the grass ovary, ovule and embryo without damaging the seed, and transmit themselves vertically from one host generation to the next. Indeed, some Epichloë exhibit choke disease on some tillers, and seed colonization on other tillers. Thus, these species simultaneously undergo sexual recombination combined with horizontal transmission between hosts, and clonal reproduction coupled with vertical transmission from one host generation to the next. Still other species do not reproduce sexually, and transmission to the next generation is restricted to vertical transmission (Schardl 2010).

The most recent taxonomic revision of this genus (Leuchtmann et al. 2014) shows that it comprises 34 species, 3 subspecies, and 6 varieties (i.e., 43 distinct lineages). Whether these species reproduce: only sexually ( 2 species plus 1 subspecies), only asexually ( 23 species plus 5 varieties), or both sexually and asexually ( 9 species plus 2 subspecies) largely determines their mode(s) of transmission into the next generation. Sexual reproduction in these species provides the ability for horizontal transmission of the fungus from one individual host plant to another. 


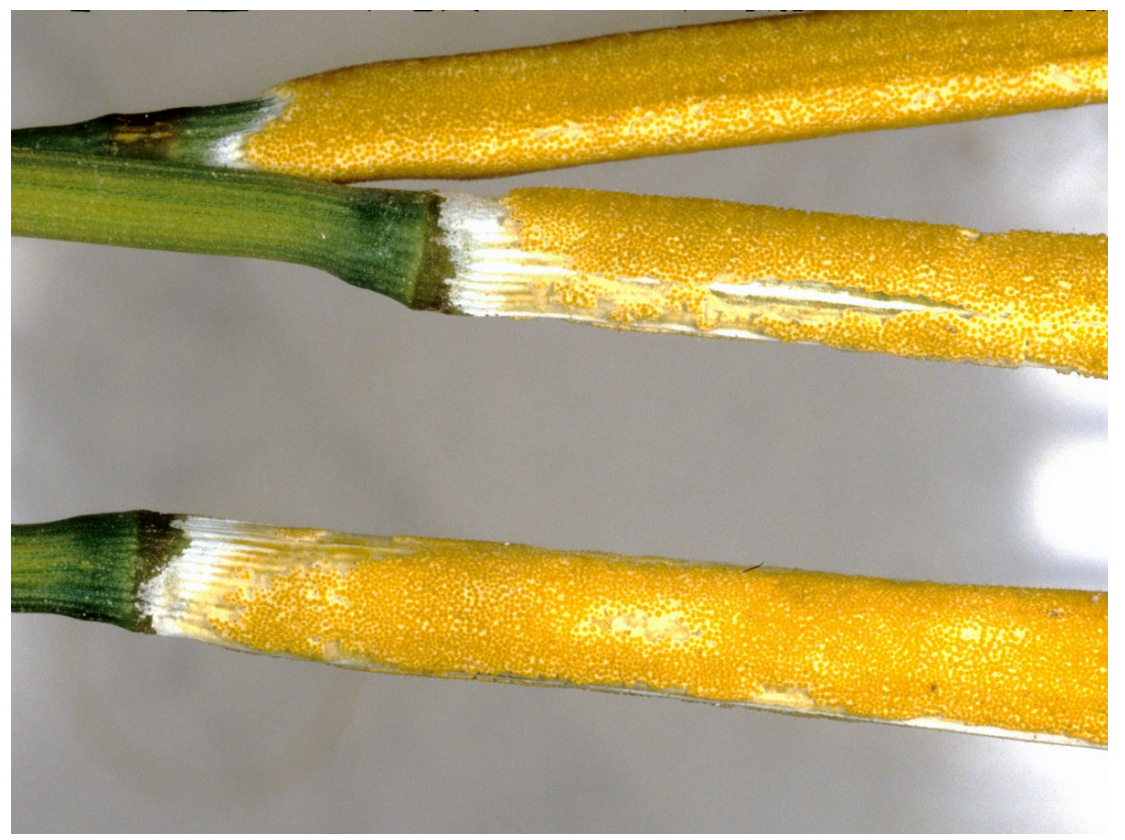

Figure 1: Epichloë typhina stromata on grass host, a condition known as 'choke disease.' Photo copyright 2013 by George Baron; used here with permission. http://hdl.handle.net/10214/5750.

Strictly asexually reproducing species seem to be limited exclusively to vertical transmission into the next generation. See Table 1 for details.

\subsection{Parasitism versus mutualism}

Sexual reproduction often involves the production of a stromatal stage that 'chokes' some of the host plant's reproductive tillers (see Fig. 1). It is often presumed that these interactions are parasitic because of the obvious fitness loss suffered by the host plant. However, most, if not all of the Epichloë lineages produce secondary metabolites, alkaloids, that can defend the host plant against some herbivores and pathogens (see, e.g., Schardl et al. 2013). So it is unclear what the balance of lifetime costs and benefits is in these sexually reproducing lineages. Thus, it is an open question as to whether these lineages are parasites or mutualists, and whether they may be either or both in different environments.

As previously mentioned, the asexual species seem to be restricted to vertical transmission. Endosymbionts that are strictly vertically transmitted ought to evolve into mutualists if they are to maintain themselves in the population (see Section 1.3). Indeed, benefits accruing to the host plants were demonstrated 'early and often' (for review of the early literature see, e.g., Saikkonen et al. 1998), and the interaction was widely asserted to be a mutualism. This view started to be challenged in the mid-1990s when Stan Faeth and his students began studying the native grass Festuca arizonica and its asexual endophyte Neotyphodium starii. ${ }^{10}$ From early on in their research, Faeth and his colleagues commonly failed to find the widely reported benefits to the host plants of the endophyte infection (see, e.g., Lopez, Faeth, and Miller 1995) and indeed found costs of harboring the endophyte. The lack of agreement needed an explanation, and one possible reason for the difference that Faeth and colleagues hit upon early on was that they

1. Note that all of the asexual Epichloë were classified as Neotyphodium until 2014. Note as well that, as of the taxonomic revision in 2014, Neotyphodium starii is no longer considered an epichloid species (Leuchtmann et al. 2014). It nevertheless is an asexual fungal endophyte that has no apparent horizontal transmission and produces alkaloids in the same classes as the Epichloë. 
Table 1: Constructed from information contained in Leuchtmann et al. (2014). Shown are the 34 species, 3 subspecies and 6 varieties of Epichloë species, cross classified by whether they are teleomorphic-types or anamorphic-types, and whether they are the result of interspecific hybridization or not. For each taxon, we indicate the presence of sexual reproduction and/or vertical transmission with $\checkmark$. The $\checkmark$ indicates that the reproduction mode occurs on some of the known hosts but not all (e.g., E. bromicola transmits vertically on 6 of the known host species, but apparently not in the other two). The + indicates that there may be multiple hosts from the same genus or that one of the hosts is known only to genus level. 'not obs.' indicates that the transmission mode has not been observed. The bold denotes taxa formerly classified in the genus Neotyphodium. See supplementary Table 1 in Leuchtmann et al. (2014) for further details.

\begin{tabular}{|c|c|c|c|c|c|c|c|c|}
\hline & Non-Hybrid & Sexual & Vertical & Hosts & Hybrid & Sexual & Vertical & Hosts \\
\hline 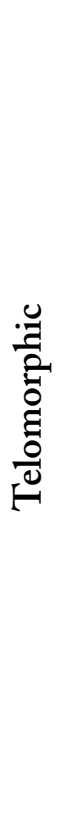 & $\begin{array}{l}\text { E. amarillans } \\
\text { E. baconii } \\
\text { E. brachyelytri } \\
\text { E. bromicola } \\
\text { E. elymi } \\
\text { E. festucae } \\
\text { var lolii } \\
\text { E. glyceriae } \\
\text { E. sylvatica } \\
\text { sub polliinensis } \\
\text { E. typhina } \\
\text { var ammophilae } \\
\text { sub clarkii } \\
\text { sub poae } \\
\text { var aonikenkana } \\
\text { var canariensis } \\
\text { var huerfana }\end{array}$ & $\begin{array}{c}\checkmark \\
\checkmark \\
\checkmark \\
\checkmark \\
\checkmark \\
\checkmark \\
\text { not obs. } \\
\checkmark \\
\checkmark \\
\checkmark \\
\checkmark \\
\text { not obs. } \\
\checkmark \\
\checkmark \\
\text { not obs. } \\
\text { not obs. } \\
\text { not obs. }\end{array}$ & $\begin{array}{c}\checkmark \\
\text { absent } \\
\checkmark \\
\checkmark \\
\checkmark \\
\checkmark \\
\checkmark \\
\text { absent } \\
\checkmark \\
\checkmark \\
\checkmark \\
\text { not obs. } \\
\text { absent } \\
\checkmark \\
\checkmark \\
\checkmark \\
\checkmark\end{array}$ & $\begin{array}{c}6 \\
5 \\
1 \\
8 \\
2+ \\
2+ \\
1 \\
1+ \\
2 \\
1 \\
10 \\
1 \\
1 \\
4 \\
1 \\
1 \\
1\end{array}$ & E. liyangensis & $\checkmark$ & $\checkmark$ & 1 \\
\hline 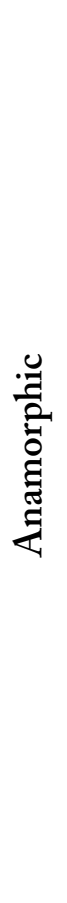 & $\begin{array}{l}\text { E. aotearoae } \\
\text { E. gansuensis } \\
\text { var inebrians } \\
\text { E. mollis } \\
\text { E. sibirica } \\
\text { E. stromatolonga }\end{array}$ & $\begin{array}{c}\text { not obs. } \\
\text { not obs. } \\
\text { not obs. } \\
\checkmark \\
\text { not obs. } \\
\text { not obs. }\end{array}$ & $\begin{array}{l}\checkmark \\
\checkmark \\
\checkmark \\
\checkmark \\
\checkmark \\
\checkmark\end{array}$ & $\begin{array}{l}1 \\
3 \\
1 \\
1 \\
1 \\
1\end{array}$ & $\begin{array}{l}\text { E. australiensis } \\
\text { E. cabralii } \\
\text { E. canadensis } \\
\text { E. chisosa } \\
\text { E. coenophiala } \\
\text { E. danica } \\
\text { E. disjuncta } \\
\text { E. funkii } \\
\text { E. guerinii } \\
\text { E. hordelymi } \\
\text { E. melicicola } \\
\text { E. occultans } \\
\text { E. pampeana } \\
\text { E. schardlii } \\
\text { E. siegelii } \\
\text { E. sinica } \\
\text { E. sinofestucae } \\
\text { E. tembladerae } \\
\text { E. uncinata }\end{array}$ & $\begin{array}{l}\text { not obs. } \\
\text { not obs. } \\
\text { not obs. } \\
\text { not obs. } \\
\text { not obs. } \\
\text { not obs. } \\
\text { not obs. } \\
\text { not obs. } \\
\text { not obs. } \\
\text { not obs. } \\
\text { not obs. } \\
\text { not obs. } \\
\text { not obs. } \\
\text { not obs. } \\
\text { not obs. } \\
\text { not obs. } \\
\text { not obs. } \\
\text { not obs. } \\
\text { not obs. }\end{array}$ & $\begin{array}{l}\checkmark \\
\checkmark \\
\checkmark \\
\checkmark \\
\checkmark \\
\checkmark \\
\checkmark \\
\checkmark \\
\checkmark \\
\checkmark \\
\checkmark \\
\checkmark \\
\checkmark \\
\checkmark \\
\checkmark \\
\checkmark \\
\checkmark \\
\checkmark \\
\checkmark\end{array}$ & $\begin{array}{c}1 \\
1 \\
1 \\
1 \\
1 \\
1 \\
1 \\
1 \\
2 \\
1 \\
2 \\
1+ \\
1 \\
1 \\
1 \\
1 \\
1 \\
12 \\
1\end{array}$ \\
\hline
\end{tabular}


were studying a 'native' grass-endophyte pair in natural settings, while much of the early work on the epichloids in general was done on introduced agricultural grasses paired with their nonnative Epichloë species in managed agroecosystems. Indeed, one can see hints of this explanation forming in the last lines of Faeth's earliest paper on the subject:

Our results, however, suggest the effect on herbivores may not be as widespread as currently believed (Clay, Hardy, and Hammond 1985; Carrol 1988; Strong 1988), or the effects are more variable in native grass-herbivore interactions. (Lopez, Faeth, and Miller 1995, 1579, emphasis added)

As Faeth's body of work began to grow in this field, seemingly so did his conviction that what he and his students were seeing was not the mutualism predicted by evolutionary theory and supported by empirical evidence in the agricultural systems. He began publishing papers with provocative titles such as:

“Neotyphodium in Native Populations of Arizona Fescue: A Nonmutualist?" (Faeth et al. 1997),

“Neotyphodium in Arizona Fescue: A Necessary Symbiont?" (Sullivan and Faeth 1999),

"What Maintains High Levels of Neotyphodium Endophytes in Native Grasses? A Dissenting View and Alternative Hypotheses" (Faeth, Sullivan, and Hamilton 2000),

"Fungal Endophytes: Common Host Plant Symbionts but Uncommon Mutualists" (Faeth and Fagan 2002),

“Are Endophytic Fungi Defensive Plant Mutualists?” (Faeth 2002),

culminating in the particularly assertive:

"Mutualistic Asexual Endophytes in a Native Grass Are Usually Parasitic" (Faeth and Sullivan 2003).

He continued to develop these ideas throughout these papers, for example:

Endophytic fungi, especially asexual, systemic endophytes in grasses, are generally viewed as plant mutualists, mainly through the action of mycotoxins, such as alkaloids in infected grasses, which protect the host plant from herbivores. Most of the evidence for the defensive mutualism concept is derived from studies of agronomic grass cultivars, which may be atypical of many endophyte-host interactions. I argue that endophytes in native plants, even asexual, seed-borne ones, rarely act as defensive mutualists. In contrast to domesticated grasses where infection frequencies of highly toxic plants often approach $100 \%$, natural grass populations are usually mosaics of uninfected and infected plants. The latter, however, usually vary enormously in alkaloid levels, from none to levels that may affect herbivores. This variation may result from diverse endophyte and host genotypic combinations that are maintained by changing selective pressures, such as competition, herbivory and abiotic factors. Other processes, such as spatial structuring of host populations and endophytes that act as reproductive parasites of their hosts, may maintain infection levels of seed-borne endophytes in natural populations, without the endophyte acting as a mutualist. (Faeth 2002, 25)²

2. Nota bene, Faeth makes reference to a 'defensive mutualism.' Most other authors do as well, because of the production of alkaloids by the endophyte, which we know deter some vertebrate and invertebrate herbivores (e.g., 
As Faeth has continued to add to this argument through the years, others too started detecting costs of the endophyte to the host grass (e.g., Cheplick, Perera, and Koulouris 2000; Cheplick 2007, 2008; Härri, Krauss, and Müller 2008a, 2008b; Fuchs et al. 2013). Taken together, Faeth's arguments and the publication of other papers demonstrating costs have given rise to the (albeit minority) view that the strictly asexual vertically transmitted Epichloë-grass interactions form a 'mutualism-parasitism continuum' (Müller and Krauss 2005). In a systematic survey of the literature (323 papers, see Appendix A), we found that for strictly vertically transmitted Epichloë species, 58\% of the papers considered the interaction to be a mutualism, but $30 \%$ considered it at least possible that the interaction could indeed be a parasitism (see Table 2 for details). And this view persists today (see, e.g., Raman and Suryanarayanan 2017).

Table 2: Summary of a systematic review of 323 papers (see Appendix A for details). We classified papers by the relationship claimed by the authors and cross-classified these claims by the mode of transmission for the Epichloë species being studied in each case (see Table 1). HT: horizontal transmission, VT: vertical transmission. Continuum denotes a claim by the authors that the Epichloe species and host plant interaction is best described as belonging on a continuum between parasitic and mutualist. Admit the possibility denotes a claim by the author that it is possible that the species interaction could be parasitic. Not stated denotes that the authors made no statement at all as to the nature of the interspecific interaction. The cells with red text represent classification errors. As we show elsewhere in this paper, it is incorrect to classify species that possess horizontal transmission as strictly mutualist (they may be, they also may be parasites). Conversely, it is incorrect to classify strictly vertically transmitted species as anything other than mutualists. See Discussion and Appendix A for more details.

\begin{tabular}{lcc}
\hline Relationship Claimed & HT or $\mathrm{HT}+\mathrm{VT}$ & $\mathrm{VT}$ only \\
\hline Parasitic & 3 & 2 \\
Continuum & 14 & 40 \\
Admit the possibility & 6 & 20 \\
Mutualism & 80 & 121 \\
Not stated & 12 & 25 \\
\hline
\end{tabular}

\subsection{Why is this view controversial?}

Cases of a parasite exhibiting only vertical transmission are interesting from an evolutionary perspective. To see why, let: $\rho$ be the proportional change in host fecundity relative to an uninfected host; $\psi$ be the proportional change in the probability of survival from seed through to reproduction; $\gamma$ be the probability an infected mother will infect an average seed; and $\delta$ be the same probability for the father. Then, from the Fundamental Vertical Transmission

Hunt and Newman 2005). Nevertheless, we know that the endophyte has many impacts on the host, prompting Rasmussen, Parsons, Popay, et al. (2008) to ask whether there was more to this mutualism than just alkaloids? We know from metabolomic and proteomic studies that these endophytes have profound effects on the plant's metabolism, biochemistry (see, e.g., Rasmussen, Parsons, Fraser, et al. 2008; Rasmussen et al. 2007; Patchett and Newman 2021; Geddes-McAlister et al. 2020) and nutrient uptake (see, e.g., Soto-Barajas et al. 2016, and references therein). Another empirically well-supported advantage conferred by the endophyte is drought resistance (see, e.g., Hosseini et al. 2016, and references therein). Our model (developed below) is agnostic about the particular mechanism(s) that leads to an endophyte advantage. Conceptually, it does not matter whether the endophyte advantage is derived from defensive alkaloids, a reduction in 'plant quality' (from the herbivore's perspective), better nutrient uptake, or drought resistance. 
Equation, it can be shown that a sufficient condition for the persistence of a parasite exhibiting only vertical transmission is $\rho \psi(\gamma+\delta)>1$, and that outside of the possibly rare condition that $\gamma+\delta>1.65$, it is also a necessary condition (Fine 1975). By definition, a parasite reduces the lifetime fitness of its host relative to an uninfected conspecific, so $\rho \psi<1$. The condition for a vertically transmitted parasite to maintain itself in a population thus requires a sufficiently high transmission rate $(\gamma+\delta)$ to counterbalance the fitness detriment it causes to its host. In the case of the Epichloë species, the fungus has never been shown to be transmitted via the pollen, and therefore $\delta=0$. Even assuming maternal vertical transmission were perfect (i.e., $\gamma=1$ ) it is not possible for the fungus to maintain itself in the population while still causing a reduction in the lifetime fitness of the host species. ${ }^{3}$ That is, the fungus cannot continue to be a parasite, and evolutionary theory thus strongly suggests that such species must evolve into mutualists (Ewald 1987).

In light of such theory, those who would argue that these strictly vertically transmitted fungi are not mutualists must provide a mechanism that explains how the fungus is maintained within a plant population, in the face of lifetime fitness costs to the plant. Faeth and Sullivan (2003) offer four possible explanations for their claim that "mutualistic asexual endophytes in a native grass are usually parasitic" (see also Faeth 2010). We briefly summarize these here. See Faeth and Sullivan for a more detailed presentation.

1. Mutualistic outcomes are rare but important. This explanation is an acknowledgement that it is lifetime fitness that matters, not a component of fitness (e.g., seed production), measured over a period of a few months or years. These cool-season perennial grasses can live for tens of years. What happens during a single season, or indeed multiple seasons, matters but has to be viewed in the context of the entire lifetime. This is particularly true if the mutualist advantage is very large in these 'rare' years. This defense amounts to an admission that the interaction is indeed a mutualism, when viewed over the whole lifetime of the host plants. We agree that this is a plausible explanation of experimental demonstrations of endophyte induced fitness reductions to the host plant. However, we feel it is misleading to refer to such a situation as a 'parasitism' when it is plainly a mutualism. In our view, it is better to refer to such observations as 'costs.'

2. Interactions vary with spatial structuring. This defense imagines a landscape containing multiple local populations of the grass hosts. In these populations, some individuals will be infected with the endophyte, and some individuals will be endophyte-free. Furthermore, it is imagined, there will be random 'catastrophes' that temporarily wipe-out some populations, and these now 'empty' patches become available for recolonization. If these local populations were closed (i.e., no dispersal between them), and the endophyte were parasitic, the whole meta-population would eventually lose the endophyte. However, if dispersal between populations is sufficiently high, and catastrophes are sufficiently common, then it is (mathematically) possible for a parasitic strain of the endophyte to persist in the meta-population (Saikkonen, Ion, and Gyllenberg 2002). Intuitively, this works because host plants infected with a parasitic strain of the endophyte would be able to disperse and recolonize empty patches (wiped out by the catastrophes) before they are out-competed by endophyte-free plants. This meta-population explanation requires significant dispersal of seeds (not pollen) between populations. With some notable excep-

3. To illustrate this point, suppose that the fitness costs of harbouring the endophyte were very small, say, $\rho=\psi=0.99999$. This means that infected hosts experience a $0.001 \%$ reduction in fecundity and survival relative to the uninfected host. Now, since $\delta=0$, even assuming that $\gamma=1, \rho \psi(\gamma+\delta) \ngtr 1$ because $0.99999 \times 0.99999(1+$ $0)=0.99998 \ngtr 1$.

๑ OPEN ACCESS - PTPBIO.ORG 
tions, grass seeds are not known for their long-distance dispersal, typically on the order of tens of centimeters (e.g., Rabinowitz and Rapp 1981), rather than the longer distances that might be necessary for persistence in a landscape such as is imagined here. Therefore, we do not think that this mechanism seems very plausible, but admittedly the hypothesis awaits experimental evidence one way or the other.

3. Asexual endophytes are transmitted horizontally. This hypothesis does not explain how strictly vertically transmitted endophytes can be parasites and still persist in the population. Instead, it changes the question. If these asexual fungal endophytes occasionally do transmit themselves horizontally, this may account for how an endophyte might be parasitic and still maintain itself in the population. This explanation seems unlikely since horizontal transmission in these species has not been observed, and, as Faeth and Sullivan admit, the horizontal transmission rate would have to be high enough to overcome the endophyte loss rate, which can be high (Afkhami and Rudgers 2008). To these caveats, we would add that basic epidemiological theory requires the dispersal rate to be high enough' to overcome the fitness detriment caused to the host plant (i.e., the parasite must be able to disperse faster than it is killing its host; see, e.g., Anderson and May 1992, Chapter 4). See also Faeth, Hadeler, and Thieme (2007) for a theoretical treatment of this idea and other variations on the theme.

4. Asexual endophytes retain control over host plant reproduction. This explanation is an attempt to extend a hypothesis about Wolbachia bacteria to the Epichloë context. Wolbachia form associations with many arthropods and filarial nematodes. In the nematodes, Wolbachia form stable mutualistic symbioses. In insects, Wolbachia are predominantly vertically transmitted, and are supposedly an evolutionary conundrum, because they seem to violate the theory, described earlier, that heritable symbionts must be mutualists. Wolbachia seem to be reproductive parasites, manipulating the reproduction of their arthropod hosts to their own advantage, and in the process often substantially decreasing the host's fitness. Wolbachia have been shown to use cytoplasmic incompatibility, killing or feminization of genetic males, and induction of thelytokous parthenogenesis. The supposed advantage of these manipulations for Wolbachia is that they are inherited solely through the female germline (as are vertically transmitted Epichloë), and their manipulations increase the proportion of females in the host population, allowing Wolbachia to spread without being a mutualist (Zug and Hammerstein 2015).

Turelli (1994) points out that, despite there being no need for Wolbachia to be a mutualist, there is still selection pressure acting to promote mutualism. A mutualist strain of Wolbachia that, in addition to manipulating its host's reproduction, also conferred a fitness advantage to the host, would spread in a population of Wolbachia where such a trait was absent. Zug and Hammerstein $(2015,90)$ point out that "recent years have seen a growing body of evidence suggesting that Wolbachia can have positive effects on the fitness of arthropod hosts and thus behave as mutualists." While the Wolbachia conundrum is still being debated, there are several elements of this story that do not seem to apply in the Epichloë case. The occasional horizontal transmission in Wolbachia, and the lack of evidence for manipulation of the grass host sex ratios are two obvious ones. t $^{-}$

4. Faeth and his students work on Festucae arizonica, which is bisexual (i.e., each flower of each individual has both male and female structures; Darbyshire and Pavlickf 2020) so it is not clear to us how the Wolbachia analogy would be applicable to this system. 


\subsection{The 'mutualism-parasitism continuum'}

Ewald (1987) probably introduced the term mutualism-parasitism continuum, but Johnson et al. seem to have popularized the term in their consideration of mychorrhizal fungi-host plant interactions. They described it thus (Johnson, Graham, and Smith 1997, 583):

Mutualism and parasitism are extremes of a dynamic continuum of species interactions. Mycorrhizal associations are generally at the mutualistic end of the continuum, but they can be parasitic when the stage of plant development or environmental conditions make costs greater than benefits, or possibly when the genotypes of the symbionts do not form 'win-win' associations. In natural systems, plant genotypes exist because they successfully propagate more plants, and fungal genotypes exist because they successfully propagate more fungi. Usually, by living together, plants and mycorrhizal fungi improve each other's probability for survival and reproductive success. But sometimes, and being anthropomorphic, plant 'interests' are in conflict with those of the fungi. In the words of Dawkins (1978) "we must expect lies and deceit, and selfish exploitation of communication to arise whenever the interests of the genes of different individuals diverge." The 'interests' of plants and mycorrhizal fungi are likely to diverge in highly managed agricultural systems, where fertilization eliminates shortages of soil nutrients, and plant genotypes are selected by humans and not by millennia of natural selection.

Although the argument about where on this supposed continuum these epichloid species sit had been going on for quite some time (for a sampling of this literature, see: Clay 1988, 1991; Saikkonen et al. 1999; Faeth et al. 1997; Faeth and Fagan 2002; Faeth, Sullivan, and Hamilton 2000; Faeth, Helander, and Saikkonen 2004; Faeth and Hamilton 2006; Rudgers et al. 2010; Rudgers et al. 2012), Müller and Krauss (2005) seem to have introduced the use of the term to the study of epichloids with their paper titled: "Symbiosis between grasses and asexual fungal endophytes":

The symbiosis between vertically transmitted asexual endophytic fungi and grasses is common and generally considered to be mutualistic. Recent studies have accumulated evidence of negative effects of endophytes on plant fitness, prompting a debate on the true nature of the symbiosis. Genetic factors in each of the two partners show high variability and have a range of effects (from positive to negative) on plant fitness. In addition, interacting environmental factors might modify the nature of the symbiosis. Finally, competition and multitrophic interactions among grass consumers are influenced by endophytes, and the effects of plant neighbours or consumers could feedback to affect plant fitness. We propose a mutualism-parasitism continuum for the symbiosis between asexual endophytes and grasses, which is similar to the associations between plants and mycorrbizal fungi. (450; emphasis added)

While debate over the usefulness of the concept continues in the mycorrhizal literature (see, e.g., Smith and Smith 2013), in this paper we argue that the concept is useful to the Epichloë situation only when discussing the genus as a whole, or when discussing individual species that possess horizontal transmission. We argue that, contra Müller and Krauss, the term is not appropriate for use with strictly vertically transmitted Epichloë. There are important biological differences between mycorrhizal fungi and Epichloë fungi that make this continuum concept less universal than it is perhaps in the mycorrhizal situation. As Ewald (1987) pointed out, transmission mode is probably the most important difference. In the next section we develop a simple model 
of each transmission strategy and use this model to draw conclusions about the nature of these Epichloë-grass interactions.

\section{A Model of Endophyte Transmission}

In this section we develop a simple continuous-time, ordinary non-linear differential equation model that covers each of the three cases of transmission: horizontal transmission only, vertical transmission only, or both horizontal and vertical transmission. Before we develop the model, we first take care of some housekeeping. The notation used in the model is summarized in Table 3.

\subsection{State variables}

Let $0 \leq x \leq 1$ be the proportion of a pasture that is infected with the endophyte. Let $0 \leq y \leq 1$ be the proportion of the pasture that is uninfected. At equilibrium, we denote these as $x^{*}$ and $y^{*}$. We can then define

$$
\phi^{*}=\frac{x^{*}}{x^{*}+y^{*}},
$$

as the proportion of the plants in the pasture that are infected with the endophyte at equilibrium. Note that $x+y$ need not equal 1 ; the difference simply represents bare ground.

\subsection{Density-dependence}

We assume that both birth and death rates are density dependent (e.g., Bullock, Hill, and Silvertown 1994). Let $b$ be the density-independent per capita birth rate, and $d$ be the densityindependent death rate. Note that the model we will develop lacks a stage-structure. Here, 'birth' means the rate at which individual plants successfully recruit from seeds (i.e., it includes seed and seedling mortality), while 'death' means the death of mature plants. We then model the density-dependent per capita birth and death rates, respectively, as:

$$
(1-x-y) b
$$

and

$$
(x+y) d .
$$

\subsection{Endophyte (dis)advantage}

Throughout the remainder of this paper we refer to the effects of the presence of the endophyte as either an advantage or a disadvantage to the host plant in terms of the effects the endophyte has, directly or indirectly, on the host's birth rate and death rate. It would also be appropriate to refer to these as benefits and costs of harbouring the endophyte. We use the term (dis)advantage to refer to the effect of the endophyte on the host generally, to denote circumstances in which the these effects might be positive or negative.

Let $a$ be the per capita birth rate (dis)advantage. We then model the endophyte infected proportion of the pasture's per capita birth rate as:

$$
(1-x-y) a b
$$


Table 3: Model definitions. These parameters and state variables are used in Eq. (9).

\begin{tabular}{|c|c|}
\hline Notation & Definition \\
\hline$x, x^{*}, y, y^{*}$ & $\begin{array}{l}\text { Model state variables. The proportion }(x) \text { of the pasture infected } \\
\text { by the endophyte and at equilibrium }\left(x^{*}\right) \text {, the proportion of the } \\
\text { pasture that is uninfected }(y) \text { and at equilibrium }\left(y^{*}\right) \text {. Note } x+y \\
\text { need not equal } 1 \text {, the difference being bare ground. }\end{array}$ \\
\hline$\phi^{*}$ & The equilibrium proportion of infected plants in the pasture. \\
\hline$a, a_{t}$ & $\begin{array}{l}\text { The per capita birth rate (dis)advantage. If } a<1 \text { then the endo- } \\
\text { phyte imposes a cost on the host in terms of the host's birth rate. } \\
\text { If } 1<a \text { then the endophyte confers an advantage to the host in } \\
\text { terms of its per capita birth rate. } a_{t} \text { denotes the case where the } \\
\text { strength of the birth rate (dis)advantage varies through time. }\end{array}$ \\
\hline$b$ & The per capita birth rate of the host plant. Default value: 0.1 . \\
\hline$c$ & $\begin{array}{l}\text { The per capita death rate (dis)advantage. If } c<1 \text { then the en- } \\
\text { dophyte confers an advantage in terms of the host's death rate. If } \\
1<c \text { then the endophyte imposes a cost in terms of the host's } \\
\text { death rate. }\end{array}$ \\
\hline$d$ & The per capita death rate of the host plant. Default value: 0.05 . \\
\hline$h$ & The per capita rate of horizontal transmission of the endophyte. \\
\hline$\ell$ & $\begin{array}{l}\text { The endophyte loss rate from seeds of infected plants in the next } \\
\text { generation. }\end{array}$ \\
\hline
\end{tabular}




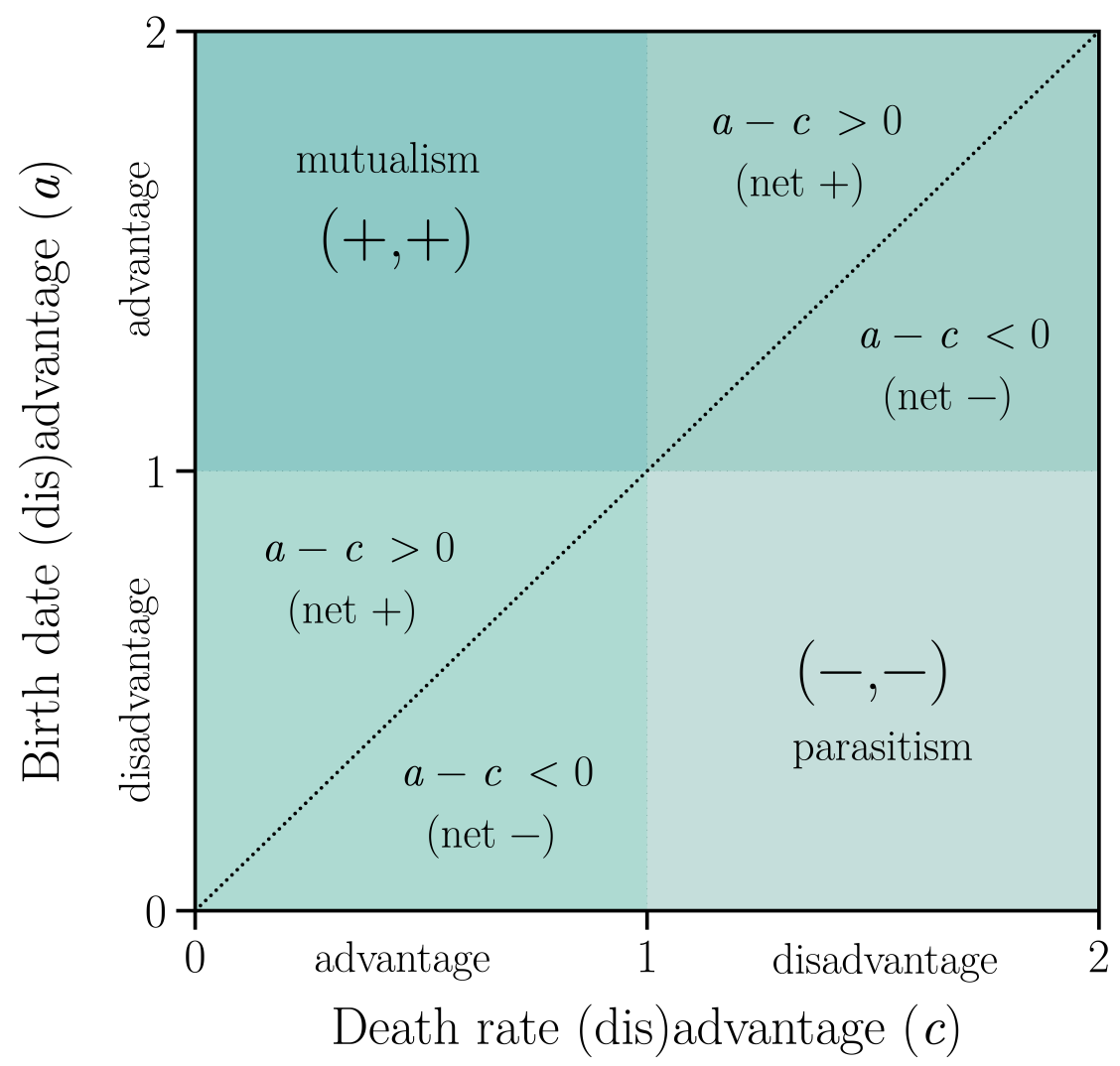

Figure 2: Parameter space. $a$ denotes the birth rate (dis)advantage. If $1<a$ the endophyte confers an advantage to the host plant in terms of the plant's birth rate. $c$ denotes the death rate (dis)advantage. If $c<1$ the endophyte confers an advantage in terms of the plant's death rate. The species interaction would certainly be called a mutualism in the upper left hand quadrant of the parameter space since the plant enjoys an advantage of the endophyte infection in terms of both its birth and death rates. In the lower right hand quadrant, the species interaction would certainly be called a parasitism since the plant suffers a disadvantage of the endophyte infection in terms of both its birth and death rates. The remaining two quadrants represent trade-offs between an advantage and a disadvantage. In the upper right the endophyte confers an advantage in terms of the plant's birth rate, but a disadvantage in terms of the plant's death rate. Whether or not this interaction is a mutualism or a parasitism depends on the relative strengths of the two (i.e., whether or not we are above the $a=c$ line or below it, respectively). And similarly for the lower left quadrant except that now the endophyte confers a disadvantage in terms of the plant's birth rate but an advantage in terms of its death rate.

If $a=1$ then there is neither an advantage nor a disadvantage to the host plant of harboring the endophyte, in terms of the plant's birth rate. If $a>1$ then the endophyte is an advantage to the plant (at least in terms of its birth rate) because it increases the birth rate. For example, if $a=1.2$ then the per capita birth rate of infected host plants will be $20 \%$ greater than the per capita birth rate of uninfected host plants. And if $a<1$ then the endophyte imposes a disadvantage on the host plant, at least in terms of its birth rate.

Similarly, let $c$ be the per capita death rate (dis)advantage. We model the endophyte infected proportion of the pasture's per capita death rate as:

$$
(x+y) c d \text {. }
$$




\section{Endophyte w/ death rate disadvantage}

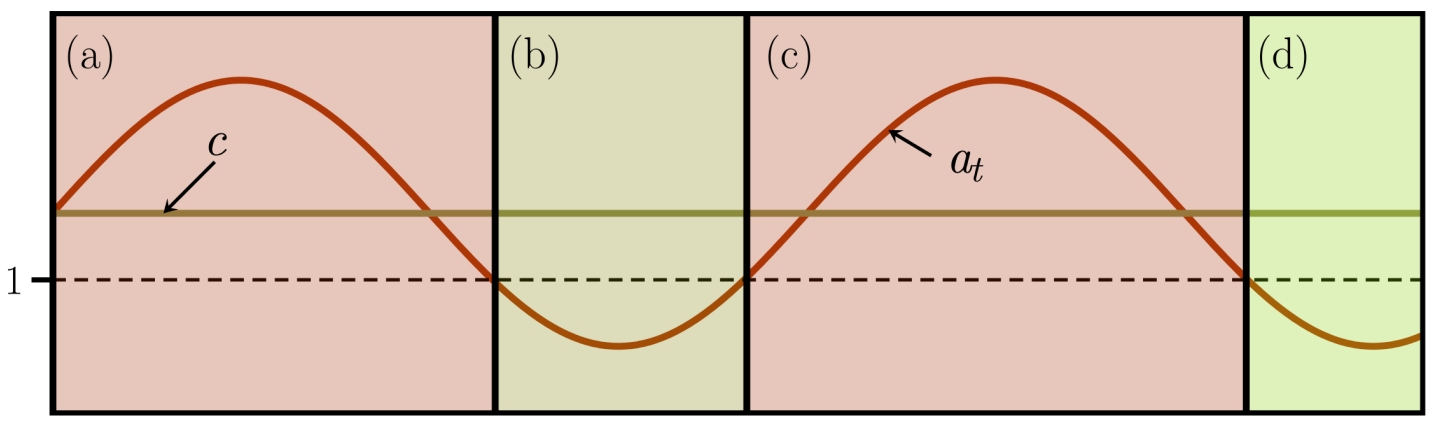

Endophyte w/ death rate advantage
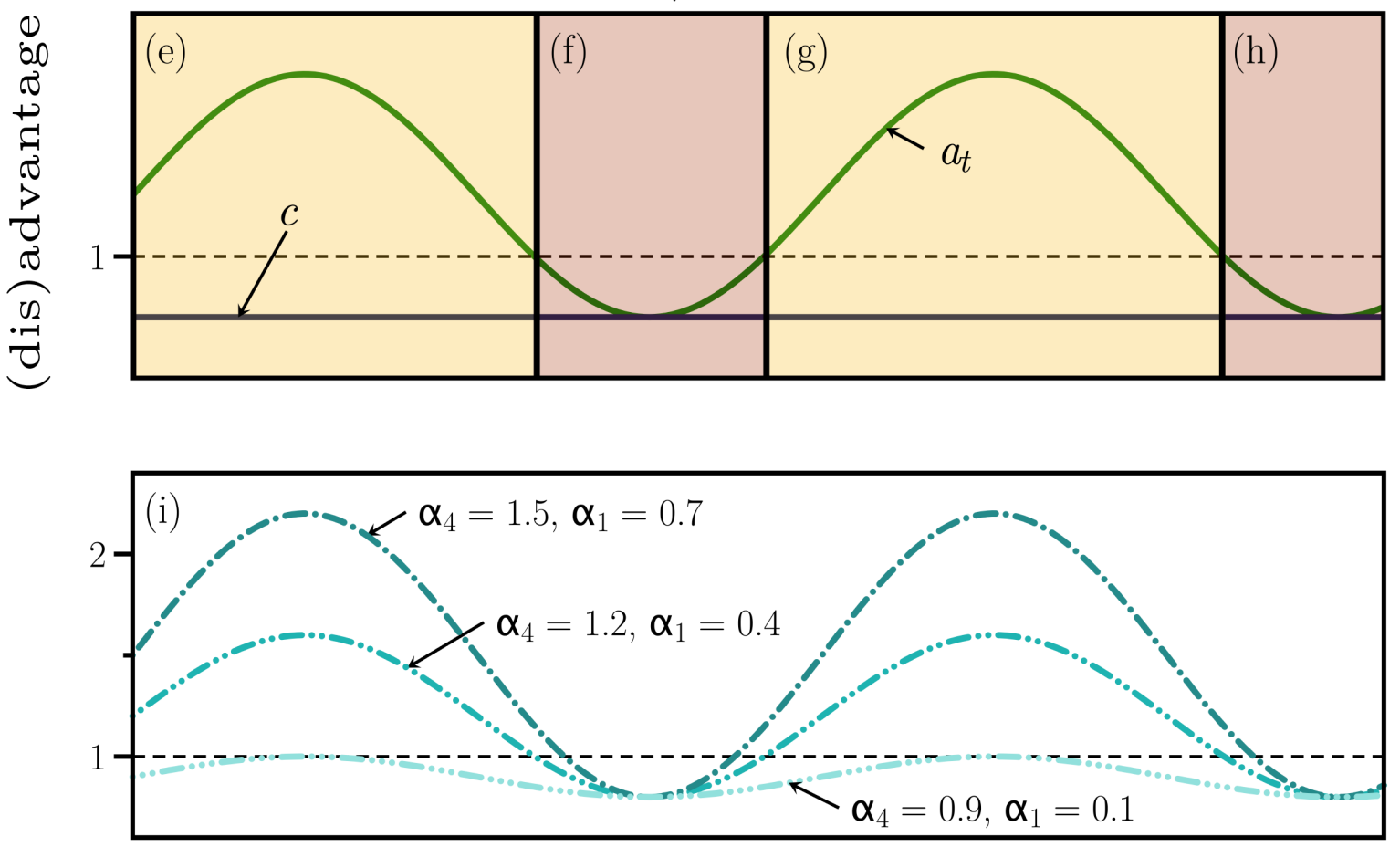

Time

Figure 3: Temporally varying birth rate (dis)advantages. The endophyte confers a birth rate advantage when $a_{t}>1$ and it confers a death rate advantage when $c<1$. The endophyte shown in $(\mathrm{a}-\mathrm{d})$ always confers a disadvantage on the host plant in terms of its death rate, while $a_{t}$ oscillates between being an advantage and a disadvantage. The endophyte shown in $(\mathrm{e}-\mathrm{h})$ always confers an advantage in terms of the host's death rate while $a_{t}$ oscillates between advantage and disadvantage. In (i) we illustrate three examples of oscillating $a_{t}$ that we use to generate the numerical results shown later.

If $c=1$ the endophyte imposes neither an advantage nor a disadvantage to the host plant. If $c<1$ then the endophyte is an advantage to the host plant, at least in terms of its per capita death rate, and if $c>1$ then the endophyte confers a disadvantage to the host plant in terms of death rates. [1 These relationships are summarized in Fig. 2.

5. Nota bene, it is important to keep in mind that $a$ and $c$ work in opposite directions. Large values of $1<a$ are advantageous because it is a multiplier of the birth rate making it larger (Eq. (4)) $)$, which is advantageous. On the other hand, large values of $1<c$ are disadvantageous because it is a multiplier of the death rate $($ Eq. (5)) $)$, making it larger, which is disadvantageous. 


\subsubsection{Temporally varying (dis)advantages}

Up to now, we have been assuming that $a$ and $c$ are constants. We think much of the confusion around the mutualism-parasitism continuum derives from situations in which there are temporally varying (dis)advantages. We therefore develop this situation as well. Let

$$
a_{t}=\alpha_{1} \sin \left(\alpha_{2}\left(t+\alpha_{3}\right)\right)+\alpha_{4} .
$$

The shape parameters work as follows. Equation 6 oscillates around $\alpha_{4} \pm \alpha_{1}$. The period of the oscillations is $2 \pi / \alpha_{2}$. The phase shift is given by $\alpha_{3}$. Note that throughout, for simplicity and without loss of generality, we let $\alpha_{3}=0$ and $\alpha_{2}=1 / 32$.

If we integrate Eq. (6) over the interval $\left[0,2 \pi / \alpha_{2}\right]$ we get:

$$
\begin{aligned}
\int_{0}^{2 \pi / \alpha_{2}} a_{t} d t & =\frac{\alpha_{1}+\alpha_{2} \alpha_{4} \frac{2 \pi}{\alpha_{2}}-\alpha_{1} \cos (2 \pi)}{\alpha_{2}} \\
& =\frac{2 \pi \alpha_{4}}{\alpha_{2}}
\end{aligned}
$$

From Eq. (7) we can show that the mean value of $a_{t}$ is:

$$
\begin{aligned}
\bar{a}_{t} & =\frac{\int_{0}^{2 \pi / \alpha_{2}} a_{t} d t}{2 \pi / \alpha_{2}}, \\
& =\alpha_{4} .
\end{aligned}
$$

One can develop a similar treatment for temporally varying $c$, but for ease of demonstration and without loss of generality, we let $c_{t}=c$ throughout.

The situation described above is illustrated in Fig. 3. How should we describe the relationships depicted in Fig. 3? Consider the endophyte described by Fig. $3 \mathrm{a} \rightarrow \mathrm{d}$, there are clearly time periods during which this endophyte is a disadvantage in terms of both the host plant's birth and death rates (Fig. 3b and 3d). Is it appropriate to refer to this endophyte as a 'parasite' during these periods? Similarly, consider the endophyte described in Fig. $3 \mathrm{e} \rightarrow \mathrm{h}$, there are times where this endophyte is an advantage in terms of both host's birth and death rates (Fig. 3e and 3g). Is it appropriate to refer to this endophyte as a 'mutualist' during these periods? For the endophyte in Fig. $3 \mathrm{a} \rightarrow \mathrm{d}$ there are also periods of time during which it is a disadvantage in terms of the host's death rate, but it is an advantage in terms of the host's birth rate (Fig. 3a and 3a). And conversely, for the endophyte depicted in Fig. $3 \mathrm{e} \rightarrow \mathrm{h}$ there are times when this endophyte confers a disadvantage in terms of the host's birth rate but an advantage in terms of the host's death rate (Fig. $3 \mathrm{f}$ and $3 \mathrm{~h}$ ). How should we describe the relationship during these periods? And finally, putting it all together, how should we describe these endophytes without regard to a specific time frame? We will return to these questions in the discussion section.

\subsection{Endophyte loss}

Let $0 \leq \ell \leq 1$ be the per capita loss rate of the endophyte, and conversely, $1-\ell$ is the transmission efficiency rate. Endophyte loss occurs through several non-mutually exclusive mechanisms. The endophyte may fail to colonize a newly produced vegetative tiller, it may fail to colonize the developing seeds prior to dispersal, and it may be lost from successfully colonized seeds prior to germination (Afkhami and Rudgers 2008). Because we are modelling individual plants, we are unconcerned with the failure of the fungus to colonize some tillers on an otherwise infected plant. We are only concerned with the failure of the fungus to give rise to a new, infected plant. 
This may happen because the seed produced is not successfully colonized prior to formation and dispersal, or the fungus may die in the seed prior to germination, or the fungal infection may fail to establish itself at the seedling stage prior to developing into a mature plant. We subsume all of these mechanisms into the single parameter $\ell$. Thus, a positive loss rate means that the proportion $\ell$ of the per capita birth rate from infected individuals will turn out to be uninfected. And $1-\ell$ will turn out to have the infection. The special case of $\ell=0$ implies that the endophyte is always perfectly transmitted to the next generation. When $\ell=1$ the endophyte is strictly horizontally transmitted.

\subsection{Horizontal transmission}

In the case of strictly horizontal transmission, we assume that all births from infected plants are initially uninfected. Furthermore, we assume that the horizontal transmission rate depends on the interactions between infected and uninfected individuals. For any encounter, the chance of passing on the infection is given by $h$ (conceptually similar to $\beta$ in standard susceptibleinfected (SI) epidemiological models; see, e.g., Anderson and May 1992). Thus the rate of horizontal transmission is given by: $h x y$. A useful extension to our model would be to relax this assumption by creating a spatially explicit model with a more mechanistically detailed treatment of horizontal transmission.

\subsection{General model}

We can use the above to construct a single model of the Epichloë-grass interaction as follows:

$$
\begin{aligned}
& \frac{\mathrm{d} x}{\mathrm{~d} t}=\underbrace{(1-\ell)(1-x-y) a_{t} b x}_{(\mathbb{1})}+\underbrace{h x y}_{(\mathcal{Q})}-\underbrace{(x+y) c d x}_{(\Re)}, \\
& \frac{\mathrm{d} y}{\mathrm{~d} t}=\underbrace{\ell(1-x-y) a_{t} b x}_{(\mathbb{4})}+\underbrace{(1-x-y) b y}_{(5)}-\underbrace{h x y}_{(2)}-\underbrace{(x+y) d y}_{(\mathbb{6})} .
\end{aligned}
$$

Here, $(\mathbb{1})$ denotes the birth rate at which new infected individuals arise from infected plants,

(2) denotes the rate of horizontal transmission by which uninfected plants become infected, (B) denotes the density-dependent death rate of infected plants, (4) denotes the birth rate at which new uninfected individuals arise from infected plants, (5) denotes the birth rate at which uninfected individuals arise from uninfected plants, and (6) denotes the density-dependent death rate of uninfected individuals. Note that if $\ell=1$ we obtain a model that represents strictly horizontal transmission, and if $h=0$ we obtain a model that represents strictly vertical transmission. Also note that if we let $a_{t}=a$ we obtain a model with temporally constant endophyte (dis)advanatges.

We next use this model to develop each of the three transmission cases separately. For each case we first develop the results for the case of $a_{t}=a$ (i.e., temporally constant birth and death (dis)advantages) and then we numerically solve for the temporally varying conditions.

\section{Case A. Strictly Horizontal Transmission, $\ell=1$}

In this first case we consider the general situation for species like Epichloë baconii, E. glyceriae, or E. typhina sub. clarkii (see Table 1). These species seem only to use horizontal transmission; vertical transmission seems to be absent. We obtain this model by setting $\ell=1$ in Eq. (9). The 


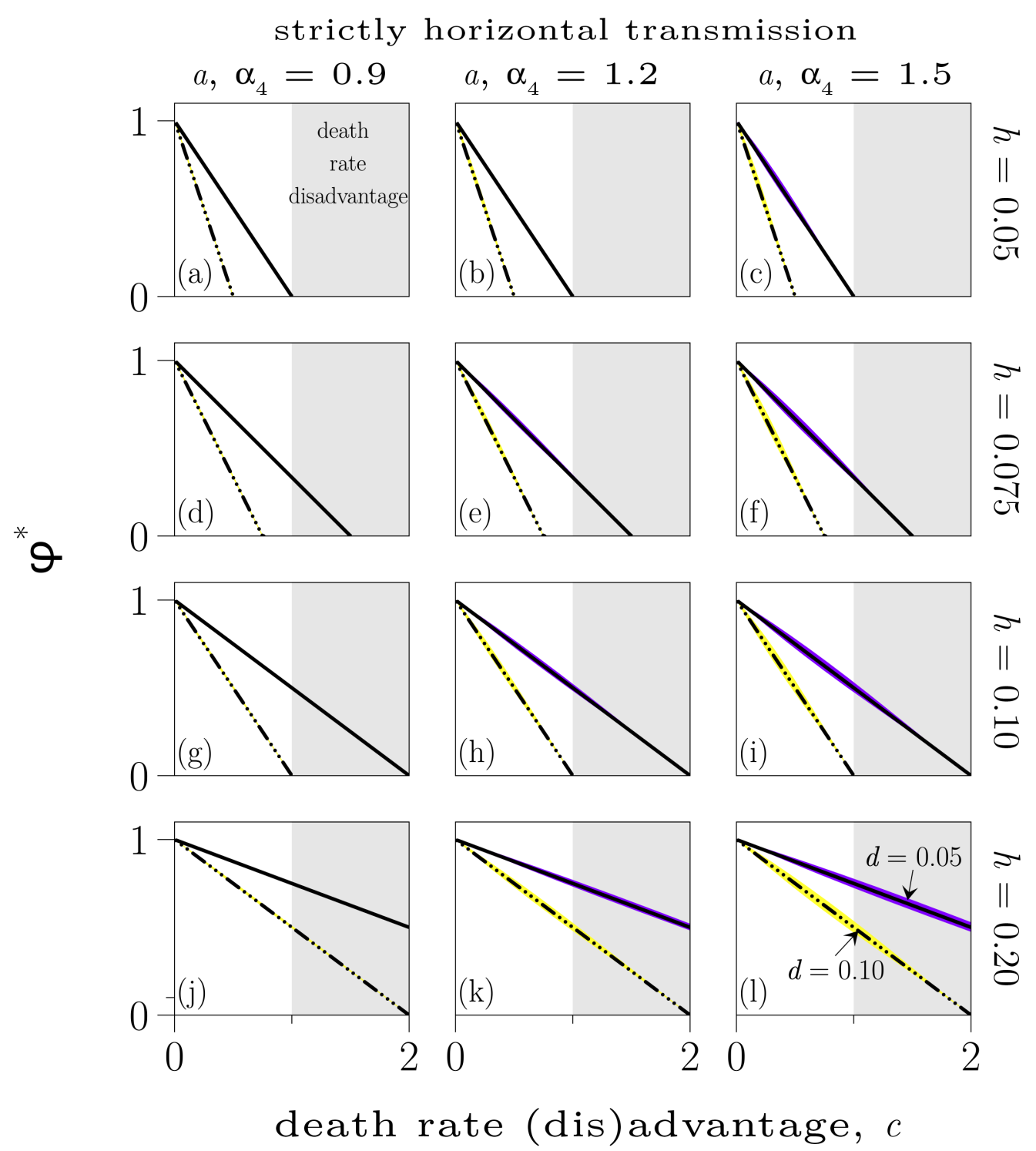

Figure 4: Equilibrium proportion of infected plants, $\phi^{*}$. Case A (strictly horizontal transmission, $\ell=1$ ); see Eq. (12). The equilibrium proportion of endophyte infected individuals in the population as a function of $c$, the death rate (dis)advantage conferred by the endophyte. Note that the equilibrium is independent of $a$, the birth rate (dis)advantage, since there is no vertical transmission in this case. $h$ denotes the rate of horizontal transmission. $d$ denotes the host plant's death rate; solid lines denote $d=0.05$, and the dash-dot lines denote $d=0.1$. Purple shading denotes the minimum and maximum values of the cyclic dynamics for Eq. (9) when $d=0.05$ and the yellow shading denotes the same when $d=0.10$.

following biological constraints exist: $0<a, 0<b, 0<c, 0<d$. Subject to these constraints, this model yields two solutions:

\subsection{Solution A.1. The pasture is totally uninfected at equilibrium}

This solution occurs in the case of $h / d<c$. In this case the infection cannot sustain itself because infected plants die faster than the infection can be passed on to susceptible plants. The equilibrium pasture densities are given by Eq. (10). Again, note that $x+y$ need not equal unity. 


$$
\left\{x^{*}=0, y^{*}=\frac{b}{b+d}\right\} .
$$

\subsection{Solution A.2. Infected and uninfected plants coexist at equilibrium}

When $c \leq h / d$, the equilibrium proportions of infected and uninfected plants in the pasture are given by Eq. (11).

$$
\begin{gathered}
\left\{x^{*}=\frac{-b(c d-h)((a-1) c d-a h)}{h(b c(d-a d)+a b h+c d(-c d+d+h))},\right. \\
\left.y^{*}=\frac{-b c d((a-1) c d-a h)}{h(b c(d-a d)+a b h+c d(-c d+d+h))}\right\} .
\end{gathered}
$$

Substituting Eq. (11) into Eq. (1) and simplifying yields:

$$
\phi^{*}=1-\frac{c d}{h} .
$$

Note that this solution is independent of any birth rate (dis)advantage. Since there is no vertical transmission, any effect the endophyte has on the host plant's birth rate only influences the birth rate of uninfected plants. Equation 12 is shown in Fig. 4. We see that the larger the value of horizontal transmission rate $(h)$ or the smaller the value of the death rate (dis)advantage $(c)$, the larger the equilibrium fraction of the pasture that is infected $\left(\phi^{*}\right)$. This matches our intuition.

This solution requires that the denominator in Eq. (11) must not be zero. Given the biological constraints on the parameters listed above, this condition is always satisfied. It can also be shown (see Appendix B) that these solutions are all stable fixed points.

\subsection{Case A. Temporal variation}

We simulated Eq. (9) for the three patterns of temporal variation in $a_{t}$ depicted in Fig. 3i. We simulated 100 years of population dynamics, which was more than sufficient to flush the initial conditions and for the system to settle into its equilibrium dynamics. These results are shown in Fig. 4 and examples of the dynamics are shown in Fig. 5a, 5d and 5g. From these figures we see that, although the equilibrium dynamics are cyclic (due to the temporally varying $a_{t}$ ) the cycles oscillate around the equilibrium from the stable point solutions found in the case where $a_{t}=a$ is a constant. We can see this more generally in Fig. 4, where the point equilibria for the constant (dis)advantage case overlay the stable cycles for the temporally varying $a_{t}$.

\section{Case B. Strictly Vertical Transmission, $h=0$}

In this case we consider those Epichloë species (see Table 1) that use only vertical transmission. In comparison to the previous case, we remove the horizontal transmission rate $(h=0)$ and we now consider that infected plants can give rise to infected plants via the vertical transmission of the fungus into the developing seeds $(0 \leq \ell<1)$.

\subsection{Case B.i. perfect transmission, $\ell=0$}

If $\ell=0$ it can be shown that the following three solutions exist. 

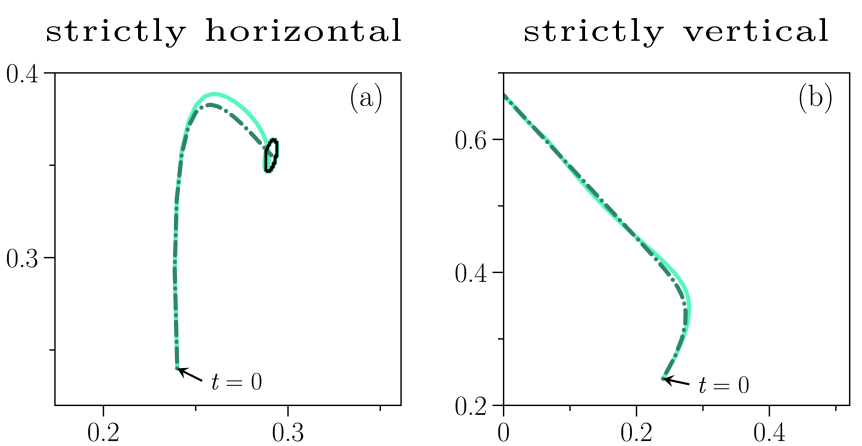

horizontal \& vertical
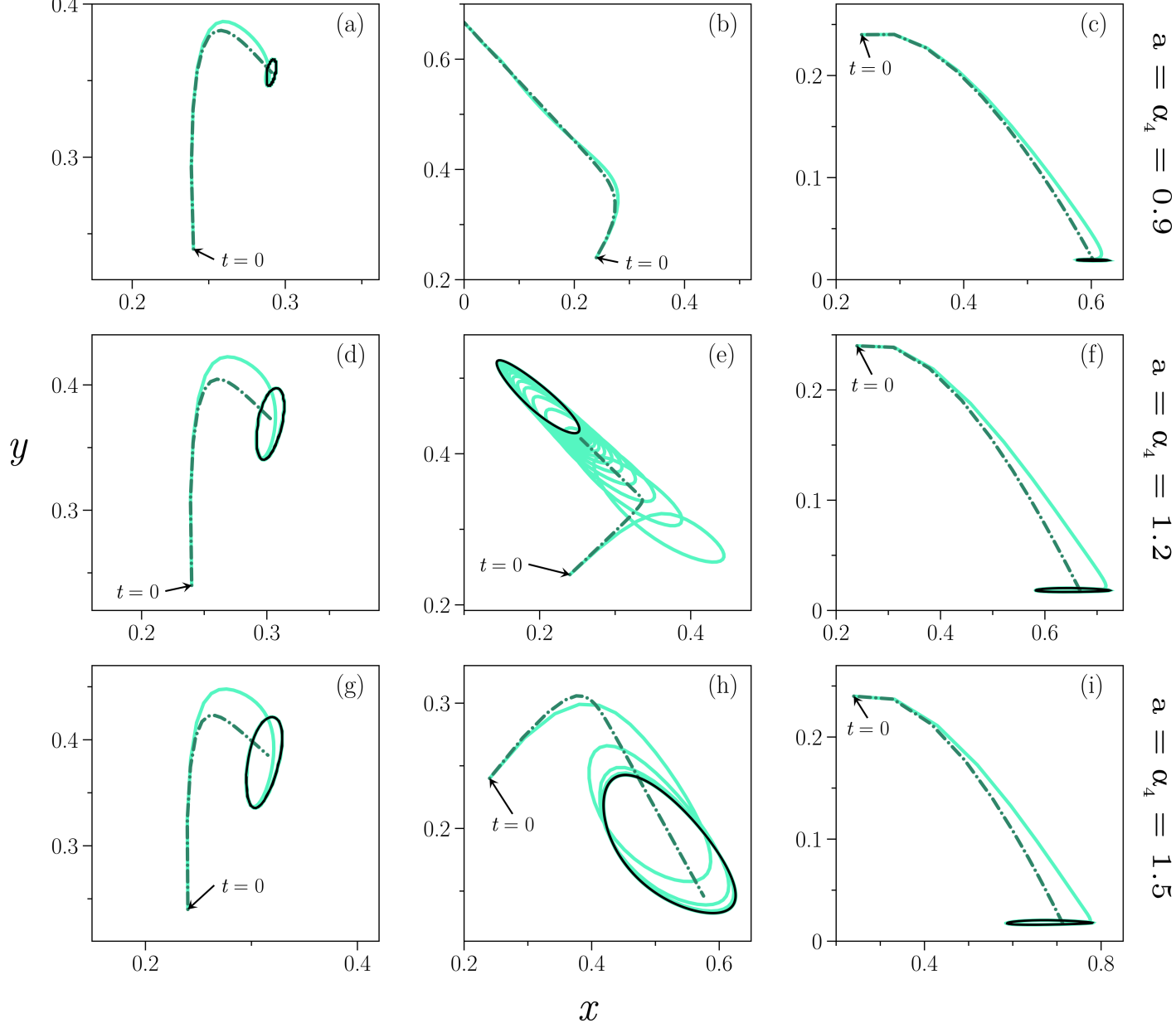

Figure 5: Phase plane diagrams of the system dynamics. Cases A and D: a strictly horizontally transmitted endophyte (i.e., $\ell=1)$ are shown in (a), (d) and (g). Cases B and D: a strictly vertically transmitted endophyte (i.e., $h=0)$ are shown in (b), (e) and (h). Cases $\mathbf{C}$ and $\mathbf{D}$ : an endophyte with both horizontal and vertical transmission are shown in (c), (f) and (i). In all of these examples, $c=1.1$ (i.e., confers a death rate disadvantage), $d=0.05$. In (a), (d) amd (g) $\ell=1$ and $h=0.1$. In (b), (e) and (h) $\ell=0.05$ and $h=0$. In (c), (f) and (i) $\ell=0.05$ and $h=0.1$. The light solid lines denote the dynamics for Eq. (9) while the dark dash-dot lines denote the corresponding dynamics for Eq. (9) where $a=\bar{a}(t)=\alpha_{4}$. The point denoted $t=0$ denotes the initial conditions. When $\alpha_{4}=0.9, \alpha_{1}=0.1$; when $\alpha_{4}=1.2, \alpha_{1}=0.4$; and when $\alpha_{4}=1.5, \alpha_{1}=0.7$; see Eq. (6).

\subsubsection{Solution B.i.1. The pasture is totally infected at equilibrium}

For $c<a$ the endophyte infection goes to fixation:

$$
\left\{x^{*}=\frac{a b}{a b+c d}, y^{*}=0\right\} \text {. }
$$

\subsubsection{Solution B.i.2. The pasture is totally uninfected at equilibrium}

For $a<c$ the endophyte infection goes locally extinct:

$$
\left\{x^{*}=0, y^{*}=\frac{b}{b+d}\right\} \text {. }
$$




\subsection{Solution B.i.3. Infected and uninfected plants coexist at equilibrium}

If, and only if, the per capita birth rate (dis)advantage equals the per capita death rate (dis)advantage, the system yields a coexistence solution-one where both infected and uninfected plants stably coexist in the population. That is, when $a=c$ we obtain:

$$
y^{*}=\frac{b-b x^{*}-d x^{*}}{b+d} .
$$

\subsection{Case B.ii. imperfect endophyte transmission, $0<\ell$}

In the case of imperfect endophyte transmission (i.e., $0<\ell$ ) the system has two just solutions.

\subsubsection{Solution B.ii.1. The pasture is totally uninfected at equilibrium}

When $0<\ell$ and $a<c$ (i.e., below the diagonal line in Fig. 2) the endophyte infection cannot be maintained in the population. We thus obtain the following equilibrium:

$$
\left\{x^{*}=0, y^{*}=\frac{b}{b+d}\right\} .
$$

This result demonstrates that when the costs of the endophyte to the host plant's fitness outweigh the benefits, the endophyte goes locally extinct. Note, this is the same equilibrium as found in Eq. (14) above.

\subsubsection{Solution B.ii.2. Infected and uninfected plants coexist at equilibrium}

When $0<\ell<(a-c) / a$ and $c<a$ infected and uninfected host plants coexist according to:

$$
\begin{gathered}
\left\{x^{*}=\frac{-a b(\ell-1)(a(\ell-1)+c)}{(a(c-1) \ell+a-c)(a b(\ell-1)-c d)},\right. \\
\left.y^{*}=\frac{a^{2} b c(\ell-1) \ell}{(a(c-1) \ell+a-c)(a b(\ell-1)-c d)}\right\} .
\end{gathered}
$$

Eq. (17) requires that $c(b+d) \neq 0$ and $b d(a(c-1) \ell+a-c)(a b(\ell-1)-c d) \neq 0$. For coexistence, given the constraints on the parameters, it can be shown that these conditions are always met (see Appendix C). Substituting Eq. (17) into Eq. (1) and simplifying yields:

$$
\phi^{*}=\frac{a-c-a \ell}{a(c-1) \ell+a-c} .
$$

Eq. (18) is plotted in Fig. 6a, 6d and 6g. There, we see that $\phi^{*} \rightarrow 0$ as $c \rightarrow a$ reflecting the constraints that $\ell<(a-c) / a$ and $c<a$. Notice that there are no solutions where both $a<1$ and $1<c$ simultaneously (Fig. 6a grey shaded area). The larger the value of $a$, the larger can be $c$ and still result in $\phi *>0$ (cf. Fig. 6d and 6g). 


\subsection{Case B. Temporal variation}

We again simulated Eq. (9) for three patterns of variation in $a_{t}$ depicted in Fig. 31. Again, we simulated the dynamics for 100 years which was sufficient to flush the initial conditions and for the system to settle into its equilibrium dynamics. These results are shown in Fig. 6a, $6 \mathrm{~d}$ and $6 \mathrm{~g}$ and examples of the dynamics are shown in Fig. 5b, 5e and 5h. Note particularly that in Fig. $6 \mathrm{a}$ and $5 \mathrm{~b}$ (i.e., the endophyte is a parasite because both $1<c$ and $\bar{a}_{t}<1$ ) the endophyte goes locally extinct, even when $a_{t}$ is not constant, demonstrating the conclusion that a strictly vertically transmitted endophyte cannot persist as a parasite. As was the conclusion for Case $\mathrm{A}$, we can see from the figures that, although the equilibrium dynamics are cyclic in the case of coexistence (due to the temporally varying $a_{t}$ ) the cycles oscillate around the equilibrium from the stable point solutions found in the case where $a_{t}=a$ is a constant. We see this more generally in Fig. 6a, 6d and 6g, where the equilibria point solutions for the constant (dis)advantage case (solid and dash-dotted line) overlay the cycles for the temporally varying $a_{t}$ (yellow and purple shading).

\section{Case C. Horizontal \& Vertical Transmission, $0 \leq \ell<1,0<h$}

In this section we consider the case for those Epichloë species that simultaneously exhibit both horizontal and vertical transmission (see Table 1).

Eq. (9) does have a close-form solution, but it is not enlightening (it involves dozens of polynomial terms). We therefore provide numerical solutions. These solutions are plotted in the second and third columns of Fig. 6. Numerically, we find that coexistence does not require mutualism. An example of such a parasitic interaction is shown in the grey shaded area (where $1<c$ ) of Fig. 6 (where $a=0.9$ ). There we see that coexistence is not only possible, but that the level of pasture infection can even be quite high, approaching $90 \%$ infection. Ceteris paribus, the higher the horizontal transmission rate $(h)$ the higher the fraction of the pasture that is infected $\left(\phi^{*} ; c f\right.$. Fig. 6b and 60; Fig. 6e and 6f; or Fig. 6h and 6i). The higher the endophyte loss rate $(\ell)$, the lower the fraction of the pasture that is infected (compare the solid and dashed-dotted lines in any panel). The lower the value of $a$ or the higher the value of $c$, the lower the equilirbium fraction of the pasture that is infected, $\phi^{*}$.

It can also be shown numerically that all of the solutions in Fig. 6 are stable fixed points; see Appendix B for more detail.

\subsection{Case C. Temporal variation}

Again, we evaluated Eq. (9) numerically for the values of $a_{t}$ shown in Fig. 3i, for a period of 100 years, which was more than adequate to flush the initial conditions and for the system to settle into its long-term dynamics. Examples of these dynamics are shown in Fig. 5c, 5f and 5i. There, we see that the solution to Eq. (9) results in a stable cycle while the solution to Eq. (9), where $a=\bar{a}_{t}$, results in a stable point around which the cyclic solutions oscillate. More generally, the results are shown in Fig. 6. Note that the solutions to Eq. (9) are cyclic, and are thus illustrated in Fig. 6 as shaded areas that denote the minimum and maximum value of the oscillations. The solutions to Eq. (9) using $a_{t}$ are nearly identical to those found using $\bar{a}_{t}$ and the same general conclusions hold.

\section{Discussion}

The model clearly demonstrates the following conclusions: 


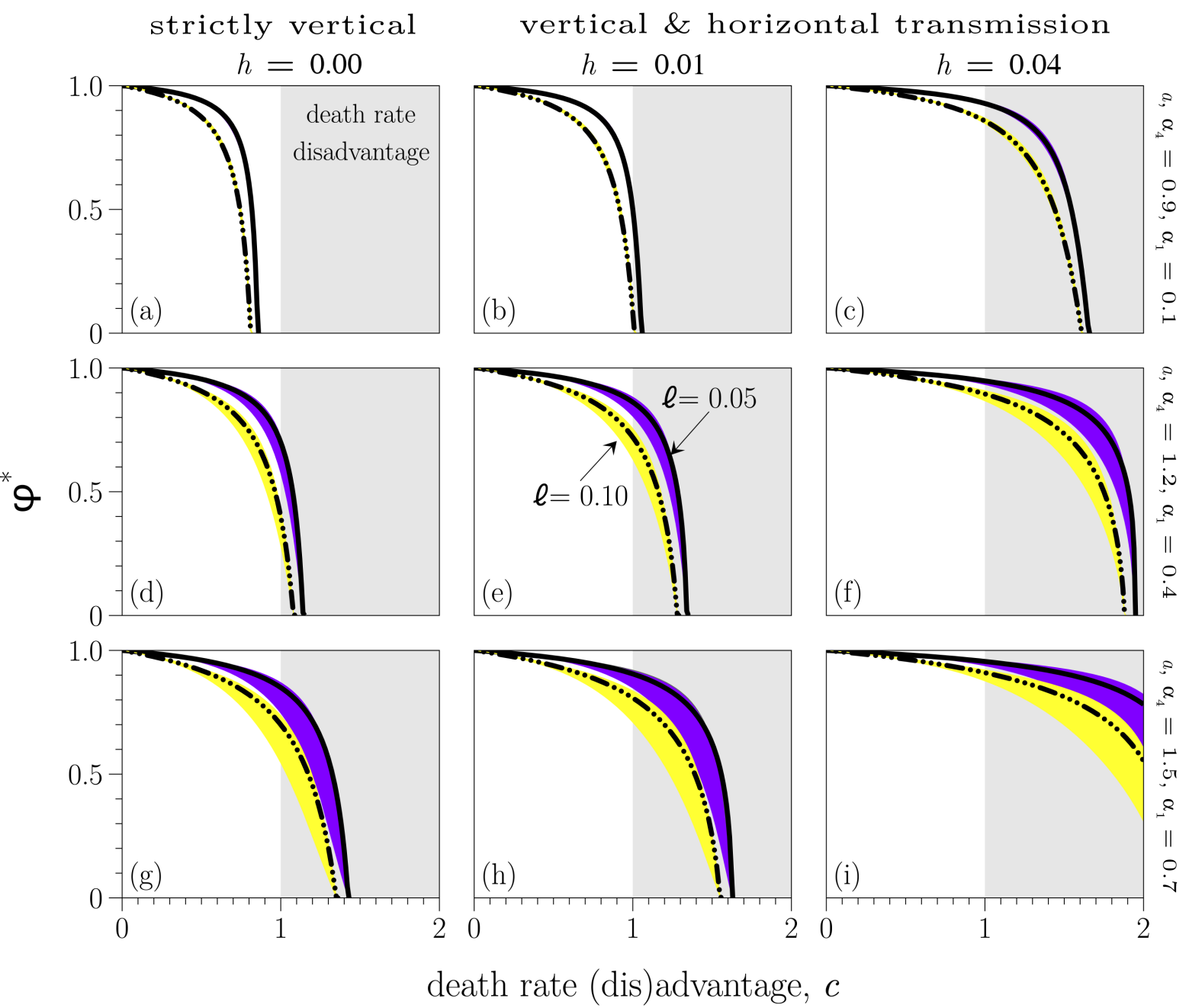

Figure 6: Equilibrium proportion of infected plants, $\phi^{*}$. Case B (strictly vertical transmission, $h=$ 0 ) is depicted in: (a), (d), and (g). Case $\mathbf{C}$ (horizontal and vertical transmission, $h>0$ ) is depicted in: (b), (c), (e), (f), (h), and (i). Case $\mathrm{D}$ (temporally varying (dis)advantages) is depicted in all panels; these results are represented by the purple $(\ell=0.05)$ and yellow $(\ell=0.10)$ shading. The shading represents the maximum and minimum values between which $\phi^{*}$ fluctuates at equilirbium (see also Fig. 5). See Eq. (6) for definitions of $\alpha_{1}$ and $\alpha_{4}$ and these are illustrated in Fig. 3i.

1. For endophytes that are strictly horizontally transmitted, the endophytes may be parasites (i.e., in this case, $1<c$ ) and still persist in the population (Fig. 4). Persistence does not require the endophyte to be a parasite (i.e., solutions in the left hand side of the graphs where $c<1$ ), but a sufficiently high horizontal transmission rate $(h)$ allows the endophyte to persist while still being a parasite $(1<c)$; see Eq. (12). In this situation it makes sense to talk about a 'mutualism-parasitism continuum.'

2. For endophytes that are strictly vertically transmitted, endophyte persistence requires that $c<a$. In other words, the advantages of endophyte infection must outweigh the disadvantages of the infection. Put differently, persistence requires that the endophyte be a mutualist. Even if we can measure disadvantages of the infection the net effect of the endophyte must be an advantage to achieve persistence ( $c f$. the grey shaded regions of Fig. 6a, 6d and 6g). And this conclusion still holds when we allow temporal variability in 
the magnitudes of the advantages and disadvantages, to include periods when there are only disadvantages $(a<1$ and $1<c$; see Fig. 6).

3. For endophytes that are both horizontally and vertically transmitted, we reach similar conclusions to those in the strictly horizontal transmission case. Sufficiently large horizontal transmission rates allow the endophyte to persist in the population while being a parasite, although there is no requirement for them to be so (see Fig. 6).

Bear these conclusions in mind as we provide the answers to the questions we asked Section 2.3.1 about Fig. 3 in the next subsection.

\subsection{Inferences from 'experimental' results}

"Thus the attitude of never believing an experiment until it's confirmed by theory has as much to be said for it as that which never believes a theory before it is confirmed by experiment." -Aris (1994, 127)

We think that the confusion surrounding the 'mutualism-parasitism continuum' is illustrated in the questions we asked earlier about Fig. 3. Here we return to those questions and provide responses. For the purposes of this discussion, imagine that the (dis)advantages shown in Fig. 3 are observations from actual plants and endophytes. Here we explore the inferences that can be drawn from such 'observations.' It is important to note that Fig. 3 does not specify the mode of transmission of the endophyte, but knowing the mode is critical to the inferences we can draw from such 'results.' The 'data' must be interpreted in light of the theoretical considerations. The two jointly determine the appropriate inferences.

\subsubsection{Question 1: Is it a parasite?}

Consider the endophyte described by Fig. $3 \mathrm{a} \rightarrow \mathrm{d}$, there are clearly time periods during which this endophyte is a disadvantage in terms of both the host plant's birth and death rates (Fig. 3b and (3d). Question 1: Is it appropriate to refer to this endophyte as a 'parasite' during these periods?

If the endophyte in question were strictly horizontally transmitted, then it would be a reasonable inference that this relationship is a parasitism. Why? Because: (i) from Fig. 4 and Eq. (12)] we know that coexistence does not depend upon $a_{t}$, only on $c$, because there is no vertical transmission and hence all new plants are uninfected; and (ii) we know from Fig. $3 \mathrm{a} \rightarrow \mathrm{d}$ that the endophyte is always a disadvantage to the plant's death rate $(1<c)$. Therefore, shortterm observations of the disadvantages, coupled with theory, suggests that inferring parasitism is reasonable. ${ }^{6}$

If the endophyte in question were strictly vertically transmitted, then an inference of parasitism would not be warranted. Why? Because: (i) the inference would be based on a relatively short (compared to the lifespan of the plant) period of time; and (ii) such an inference flies in the face of a good deal of contradictory experimental evidence about strictly vertically transmitted endophytes; and (iii) solid evolutionary theory suggests the relationship cannot be a parasitism (as we have demonstrated here, and others have demonstrated elsewhere; see Section 6.3).

6. Note that strict horizontal transmission does not require the endophyte to be a parasite, but in this case it is, by definition (i.e., $1<c$ always and $a_{t}$ is irrelevant). 
Finally, if the endophyte in question were transmitted horizontally and vertically, it would be difficult to make any inference. Why? Because: (i) theory tells us that both parasitism and mutualism are plausible outcomes of this interaction (Fig. 6), and (ii) the evidence provided by the 'observations' in Fig. 3b or $3 \mathrm{~d}$ are not sufficient by themselves to settle the question. We would need to know the net effects of the advantages minus the disadvantages intergrated over a long period of time. Here, it might be appropriate to employ the mutualism-parasitism continuum concept as a descriptor.

\subsubsection{Question 2: Is it a mutualist?}

Now consider the endophyte described in Fig. 3e $\rightarrow$ h. There are times (Fig. 3e and $3 g$ ) in which this endophyte is an advantage in terms of both host's birth and death rates. Question 2: Is it appropriate to refer to this endophyte as a 'mutualist' during these periods?

For a strictly horizontally transmitted endophyte, we could reasonably infer that this relationship is a mutualism. Why? Because: (i) theory tells us that the fluctuations in the host's birth rate (dis)advantage are irrelevant since all offspring produced are initially endophyte-free (i.e., no vertical transmission), and (ii) we know from Fig. $3 \mathrm{e} \rightarrow \mathrm{h}$ that this endophyte always produces an advantage in terms of the host's death rate.

For a strictly vertically transmitted endophyte, it would also be reasonable to infer that the endophyte is a mutualist. Why? Because: (i) we observe benefit in terms of both the host's birth and death rates, and (ii) theory tells us that strictly vertically transmitted endophytes must be mutualists to persist.

If the endophyte in question were transmitted horizontally and vertically, it would again be difficult to make any inference. Why? Because: (i) theory tells us that both parasitism and mutualism are plausible outcomes of this interaction, and (ii) the evidence provided by the 'observations' during these periods (Fig. 3e or Bg) are not sufficient by themselves to settle the question. Here again, it might be appropriate to employ the continuum concept as a label.

\subsubsection{Question 3: How should we interpret mixed (dis)advantages?}

For the endophyte in Fig. $3 \mathrm{a} \rightarrow \mathrm{d}$ there are also periods of time during which it is a disadvantage in terms of the host's death rate, but it is an advantage in terms of the host's birth rate (Fig. 3a and 3c). And conversely, for the endophyte depicted in Fig. $3 e \rightarrow h$ there are times when this endophyte confers a disadvantage in terms of the host's birth rate but an advantage in terms of the host's death rate (Fig. 3f and 3h). Question 3: How should we describe the relationship during these periods of mixed advantages and disadvantages?

If the endophyte in question were strictly horizontally transmitted, it would again be reasonable to infer that the relationship is a parasitism (Fig. 3a and 3c) or mutualism (Fig. 3f and 3h). Why? Again, because: (i) $a_{t}$ is not a relevant consideration in the case of a strictly horizontally transmitted endophyte, and (ii) we know that the endophyte is always a disadvantage $(1<c)$ in Fig. $3 \mathrm{a} \rightarrow \mathrm{d}$ and is always an advantage in Fig. $3 \mathrm{e} \rightarrow \mathrm{h}$, and (iii) theory tells us that both parasitism and mutualism are plausible outcomes of this interaction.

If the endophyte in question were strictly vertically transmitted, we might reasonably infer that the relationship is a mutualism in both cases. Why? Because: (i) integrated over the time period represented by the red shading the advantages in terms of the host's birth rate seem to outweigh the disadvantages in terms of the host's death rate (by visual inspection), and (ii) theory tells us that this relationship must be a mutualism if it is to persist. 
Finally, if the endophyte in question were transmitted horizontally and vertically it again would be difficult to make any inference. Why? Because: (i) theory tells us that both parasitism and mutualism are plausible outcomes of this interaction, and (ii) the evidence provided by the 'observations' during the red shaded periods is again, not sufficient by itself to settle the question. And again, it might be appropriate to employ the continuum concept as label.

\subsubsection{Question 4: How should we describe these interactions?}

Finally, putting it all together, Question 4: How should we describe these endophytes without regard to a specific time frame?

In the case of a strictly horizontally transmitted endophyte, in these cases, we could reasonably infer that it is a parasite in Fig. $3 \mathrm{a} \rightarrow \mathrm{d}$, and a mutualist in Fig. $3 \mathrm{e} \rightarrow \mathrm{h}$. Why? Because again: (i) $a_{t}$ is not a relevant consideration, and (ii) the endophyte always confers a disadvantage in the first instance and an advantage in the second. However, these particular conclusions rely on the artefact that we kept $c$ as a constant. More generally, if $c=c_{t}$ was temporally varying, it would be difficult for us to make any inference. This is because theory tells us that mutualism or parasitism are plausible outcomes, and we would need to know the net effect of the temporally varying death rate (dis)advantages over a long period of time. Again, it might be useful to employ the continuum concept to label this interaction, absent further convincing evidence placing the interaction at one end of the continuum or the other.

In the case of a strictly vertically transmitted endophyte, we would likely infer that the relationship is a mutualism for both endophytes Fig. $3 \mathrm{a} \rightarrow \mathrm{d}$ and Fig. $3 \mathrm{e} \rightarrow \mathrm{h}$. Notwithstanding the evidence of an intermittent net disadvantage of the endophyte in Fig. $3 \mathrm{a} \rightarrow \mathrm{d}$, theory tells us that the long-term net impact of the endophyte must be positive for the interaction to persist in a particular location. Describing the interaction shown in Fig. $3 \mathrm{a} \rightarrow \mathrm{d}$ as anything other than a mutualism would be unwarranted. Nevertheless, observing such periods of intermittent net disadvantage of an endophyte that is strictly vertically transmitted would certainly prompt follow-up studies and the search for alternative explanations (e.g., perhaps the endophyte is not strictly vertically transmitted?).

Finally, in the case of an endophyte possessing both vertical and horizontal transmission, in either case (Fig. $3 \mathrm{a} \rightarrow$ d or Fig. $3 \mathrm{e} \rightarrow \mathrm{h})$ it would be reasonable to infer that these endophytes sit somewhere along a mutualism-parasitism continuum, and that where on that continuum it sits depends on the long-term advantages minus the disadvantages. This is a reasonable inference because (i) theory tells us that both mutualism and parasitism are plausible outcomes, and (ii) the observations for both endophytes show that over time there is a mixture of advantages and disadvantages.

\subsection{Model limitations and useful future extensions}

All models are simplifications or abstractions. We think our abstraction captures the most important general mechanisms and therefore offers a reasonable approximation to the biological system it is meant to represent. Others might quibble with this assertion. Certainly there are important aspects of the biology that are not incorporated in our model. For example, there is no representation of the seed-bank dynamics. The model lacks a stage or age structure. The model also has not been explicitly parameterized for any specific host-endophyte pair, nor for any specific geographical location. By keeping the model non-specific in these ways, we think it demonstrates some useful generalizations. Whether adding any of these biological realisms affects the general conclusions remains to be seen, but our intuition is that the general requirements for coexistence will hold, at least in a qualitative way.

๑ OPEN ACCESS - PTPBIO.ORG 
One well-known mechanism by which these endophytes may confer an advantage to the host plant is through increased drought tolerance (White et al. 1992). A drought gradient has long been hypothesized to account for the distribution of endophyte-infected perennial ryegrass (Lolium perenne) in Europe (Lewis et al. 1997). We will explore this mechanism in future work.

Another useful extension, not previously considered in any rigorous way, would be to explore the role of environmental stochasticity on coexistence. There are plenty of examples in the literature in which stochastic models lead to different, sometimes deeper, understandings than the corresponding deterministic model (see, e.g., Newman 1991; Black and Mckane 2012).

\subsection{Comparisons with previous modeling work}

Ecologist Richard Levins (1966) famously stated:

However, even the most flexible models have artificial assumptions. There is always room for doubt as to whether a result depends on the essentials of a model or on the details of the simplifying assumptions ....

Therefore, we attempt to treat the same problem with several alternative models each with different simplifications but with a common biological assumption. Then, if these models, despite their different assumptions, lead to similar results we have what we can call a robust theorem which is relatively free of independent lies. (423) ...

Unlike the theory, models are restricted by technical considerations to a few components at a time, even in systems which are complex. Thus a satisfactory theory is usually a cluster of models. These models are related to each other in several ways: as coordinate alternative models for the same set of phenomena, they jointly produce robust theorems; as complementary models they can cope with different aspects of the same problem and give complementary as well as overlapping results; as hierarchically arranged "nested" models, each provides an interpretation of the sufficient parameters of the next higher level where they are taken as given. (431)

In this subsection, we review previous modelling attempts for the Epichloë-grass interaction, and compare and contrast our model to the previous work. We conclude that, taken together, this set of models produces a robust theorem about this system: that strictly vertically transmitted Epichloë endophytes must be mutualists.

Clay (1993) developed a very simple fitness-based model, without endophyte loss, to show that for strictly vertically transmitted fungal endophytes with perfect transmission efficiency, any net fitness reduction caused by the endophyte results in the loss of endophyte-infected individuals from the population, echoing earlier work (Ewald 1987; Fine 1975). Clay used this model to show that the equilibrium proportion of infected individuals in the pasture is either 0 or 1 , and the only means of obtaining a mixed solution was for the benefit of the endophyte to exactly balance the costs, a condition Clay thinks is unlikely to occur. These results are confirmed again by our work, sensu Eqs. (13) to (15). The addition that our work contributes here is that by placing our model in a population dynamics framework, we are able to demonstrate how, for this very restricted case $(a=c)$ it is possible to get other proportions in the mixture apart from the $1 / 2$ that Clay finds. We show that the particular mixed result achieved depends on the host plants' birth and death rates (Eq. (15)). 
Ravel, Michalakis, and Charmet (1997) develop a discrete time, stage structured population dynamics model. They divide the endophyte effects on the host plant into effects on birth rate, death rate, and competitive ability. They do not examine cases where the endophyte might be a disadvantage to the host plant in terms of birth and death rates, as we do, although they do briefly admit the possibility that the endophyte might leave the host plant at a competitive disadvantage. The result of this possibility, however, is never explored in their results. In our model, we do not represent competition as distinct from the birth and death rate (dis)advantages. In our model, the competitive advantage of the endophyte, if there is one, arises from how it affects the host plants' birth and death rates. Finally, Ravel et al. consider a stochastic version of their model where the birth rates, death rates, and competitive advantages are sampled each year from a normal distribution but they provide no details on the mean or variance of these normal distributions, nor do they explore how the mean and variance interact to affect the results. They merely conclude that stochastic effects are only important in small populations, something that has long been known in population ecology. Mostly, Ravel et al. use their model to explore the trade-off between the endophyte loss rate (their $\mu$, conceptually equivalent to our $\ell$ ) and the birth and death rate advantages of the endophyte. In this paper we are primarily interested in how birth and death rate advantages and disadvantages can trade-off against each other to influence the equilibrium infection rate. For us, $0<\ell$ is simply a necessary condition to obtain stable mixtures of infected and uninfected plants.

Gundel et al. (2008) modeled a closed annual grass population using a stage-structured, density-independent, periodic, non-stochastic matrix model. We used a non-stage-structured, density-dependent model of a perennial grass population. Like Gundel et al., we provided an example of an environment of deterministic seasonality (see Fig. 3). Also like Gundel et al., we assumed that the grass' vital rates $(b$ and $d)$ and transmission efficiency $(1-\ell)$ were constant through time. Gundel et al. used their model to examine how the endophyte loss rate interacts with the ratio of reproductive rates of the infected and uninfected plants to determine the equilibrium infection frequency. Echoing the earlier work, they conclude that:

This condition implies that, in a closed population, the endophyte can only persist in the long term if the infection results in some increase in the host plant fitness .... However, such an increase could be very small .... (900)

Making different assumptions, and structuring our model differently, we find the same result, the combined benefits have to outweigh the costs (i.e., there has to be an increase in the host plant's net fitness). However, we show that just because one measures a cost in terms of birth or death rates, this does not imply that the endophyte can persist in a population as a parasite. Furthermore, Gundel et al. (2008, 902) make the important point that this net fitness benefit of the endophyte can be so small that it would be difficult to measure under field conditions, yet this small difference could result in high infection frequencies in pastures. We find that this conclusion is true, but only for perfect transmission (i.e., $\ell=0$ ). Once the endophyte loss rate is positive, small net positive effects of the endophyte on the host lead to coexistence, but at a relatively low frequency of infection in the pasture.

Gundel et al. (2008, 897-8) observed that "relatively high infection frequencies have been observed in natural grass populations exhibiting little or no evidence that the fungus confers a reproductive advantage to its host (Saikkonen et al. 1998; Faeth and Hamilton 2006)." Our model provides two possible explanations for this phenomenon. First, such a solution can occur when the endophyte provides a survivorship advantage instead of a reproductive advantage. Second, if costs and benefits of endophyte infection vary within and between years, it would be possible for the pasture to still maintain a high infection rate even if in some seasons, or across 
some years, the endophyte provides no benefit, or even a short-term net cost. Notice in Fig. 6 there are examples where $a<1$, i.e., the endophyte is a disadvantage to the host in terms of reproduction, and yet the endophyte still persists in the population, even possibly at relatively high proportions of the pasture depending on the death rate (dis)advantage $(c)$.

Finally, Saikkonen, Ion, and Gyllenberg (2002) use meta-population dynamics to understand how the endophyte can be a parasite in some places and still persist on the landscape. They find that this is indeed possible so long as the seed dispersal rates are high enough, and there exist sufficient 'empty' patches into which the parasite-infected host plant can disperse. As we stated in the introduction, we do not find the meta-population dynamics explanation convincing. While long-distance dispersal of grass seeds is possible, aided by animals for instance, the vast majority of seed dispersal in these grasses is quite local. To us it seems unlikely that such restricted dispersal would be sufficient to drive the meta-population explanation. Nevertheless, our model cannot be directly compared to this model as we consider closed populations and ignore immigration and emigration.

\section{The Continuum Reconsidered}

Most (if not all) symbioses impart a cost on the host, even when the symbiosis is a mutualism, and those costs may be larger or smaller depending on the environment in which they are measured. Nevertheless, costs are not the same thing as parasitism. Suppose that the only advantage conferred by an endophyte was the production of alkaloids that deter insect-herbivores. The host plant pays a cost in terms of photosynthate to support the endophyte's metabolism, as well as a nitrogen cost for the production of the alkaloids. In such a case, the benefit of the endophyte would only be realized when there are insect-herbivores present. There will be days when there are insects present and the endophyte's benefits are greater than its costs, and days when insects are absent and the endophyte's costs are greater than its benefits. 0 What are we to conclude about the underlying nature of this grass-endophyte relationship? Some would say that this symbiosis falls on a 'mutualism-parasitism continuum.' Used in this way, the continuum concept only means that net benefits fluctuate through time, and we argue that this is misleading and obscures the true nature of the interaction.

Now, imagine this same symbiosis, but studied in two different environments, one in which insect-herbivore pressure is very high, and one in which that pressure is very low. It might make sense to say this symbiosis is on the mutualism-parasitism continuum, at the mutualism end in the former environment and at the parasitism end in the latter environment. However, we have argued in this paper, and demonstrated with our model, that it makes sense to speak about a mutualism-parasitism continuum of interspecific interactions between epichoid fungal species and their host grass species when either: (a) we are talking about the genus as a whole, or (b) we are talking about individual Epichloë species that have the ability to transmit themselves horizontally. It does not make sense to describe Epichloë species that are strictly vertically transmitted as anything other than mutualists. There may well be times when researchers are able to measure costs to the host plant of this interaction. Nevertheless, over time, those costs must be outweighed by the benefits for the fungus to persist in any population. In our hypothetical example, if the endophyte were strictly vertically transmitted, and if there was an environment that consistently had very low insect-herbivore populations, such that the net effect of the benefits-costs (advantages-disadvantages as we have used throughout this paper) of

7. This argument is the same for any time span. It applies to weeks, months, seasons, even years. Our use of 'days' is a sort of reductio ad absurdum. It makes little sense to say the symbiosis is a parasitism on Tuesday but a mutualism on Wednesday.

๑ OPEN ACCESS - PTPBIO.ORG 
the endophyte are always negative, then that endophyte would not persist in that environment. Some might argue that in such an environment the endophyte is indeed a parasite. We argue that such a classification is both wrong and misleading. At best, one might plausibly claim that in such a situation the endophyte is in an unstable, transient, or failed parasitism.

\section{Appendix A Systematic Literature Survey of Positions}

We conducted a systematic review of the literature to examine the frequency and time trajectory of positions held by authors toward the 'parasitism-mutualism continuum.' On 5 November 2019, we executed the following search in Web of Science: (Epichloë OR Neotyphodium) AND (Parasit* OR Mutuali*). This search yielded 399 papers published between 1991 and 2019. We eliminated papers that were about the mutualism between pollinating flies and the sexually reproducing Epichloë species, or that mention the fungus only superficially (e.g., only in the reference section). We also excluded papers that were not readily available. The remaining 323 papers were examined by searching the text for 'parsiti' and 'mutuali' and reading what these authors said specifically about the nature of the plant-fungal interaction. Note that some authors used 'antagonism' or 'pathogenic' (and their derivatives) to describe a 'parasitic' relationship. We searched these terms too.

We classified each paper as taking one of five possible positions: (1) 'parasitic/parasitism', (2) 'mutualistic/mutualism', (3) 'unclear or a continuum', (4) 'admits the possibility' of parasitism, or (5) 'not stated' (i.e., makes no statements about mutualism or parasitism). Number 1 was an extremely rare position (5 of 326 papers) and we suspect that the authors were using the word 'parasitism' differently than others in this field. To be classified as 'mutualism' the authors must have made statements that either the relation is a mutualism, or that it is believed to be, thought to be, etc. a mutualism. To be classified as 'unclear or a continuum' we looked for statements such as:

Symbioses between cool-season grasses (Poaceae subfamily Pooideae) and fungi of genus Epichloë (including their asexual derivatives in the genus Neotyphodium) are very widespread and occur in a broad taxonomic range of this important grass subfamily. These symbioses span the continuum from mutualistic to antagonistic interactions. (Schardl et al. 2008, 483)

The above quote clearly refers to the genus Epichloë as a whole, and is therefore a correct usage of the continuum concept (i.e., it is certainly correct that various species sit at different locations along the continuum). Here is an example of the implied use of the continuum concept when clearly speaking only of the strictly vertically transmitted species; we suggest this is an inappropriate characterization of the nature of the interaction:

The negative effect of asexual endophytes on their hosts may evolve because the reward for the partners and the ability to control the interaction is asymmetrical (Saikkonen et al. 2004). The fitness of the fungus depends strongly on the fitness of the plant (Clay and Schard1 2002), but the plant does not always benefit from the presence of the fungus (Faeth and Sullivan 2003). This, together with the fact that the host-endophyte interaction seems to be controlled by the host (Christensen,

8. Note that prior to 1991, the asexual Epichloë species were classified as Acremonium species. We chose not to survey the older literature because it pre-dates Faeth et al.'s claims of parasitism by strictly vertically transmitted fungal endophytes. 
Bennett, and Schmid 2002), makes it likely that the outcome of the interaction is antagonistic rather than mutualistic. A supposedly mutualistic host-endophyte interaction may appear parasitic after detailed investigation. Here, we show that an apparently beneficial asexual endophyte may have a negative effect on the reproduction of its host. (Olejniczak and Lembicz 2007, 486)

A paper was classified as 'admitting the possibility' if it referred to the idea that the interaction might be parasitic without appearing to endorse that position. This category might be viewed as a weaker version of the 'unclear or continuum' category. Finally, 'not stated' was used when the authors did not discuss the nature of the endophyte-plant interaction.

Fig. A.1 shows the accumulation of papers taking each of the above positions. As of 2019, of the 286 papers that take a position, $70 \%$ state that the relationship is a mutualism, and 30\% take the position that the relationship is 'unclear,' a 'continuum,' or at least it is possible the relation is not a mutualism.

These numbers are confounded with the relative publishing frequency for authors taking the various positions. However, it is not uncommon to see the same authors' positions shifting through time, including us (mutualism: Newman et al. 2003; Ryan et al. 2014; Shukla et al. 2015; Bastías et al. 2018), (unclear/continuum/admits possibility: Hunt and Newman 2005; Rasmussen et al. 2007; Antunes et al. 2008). 


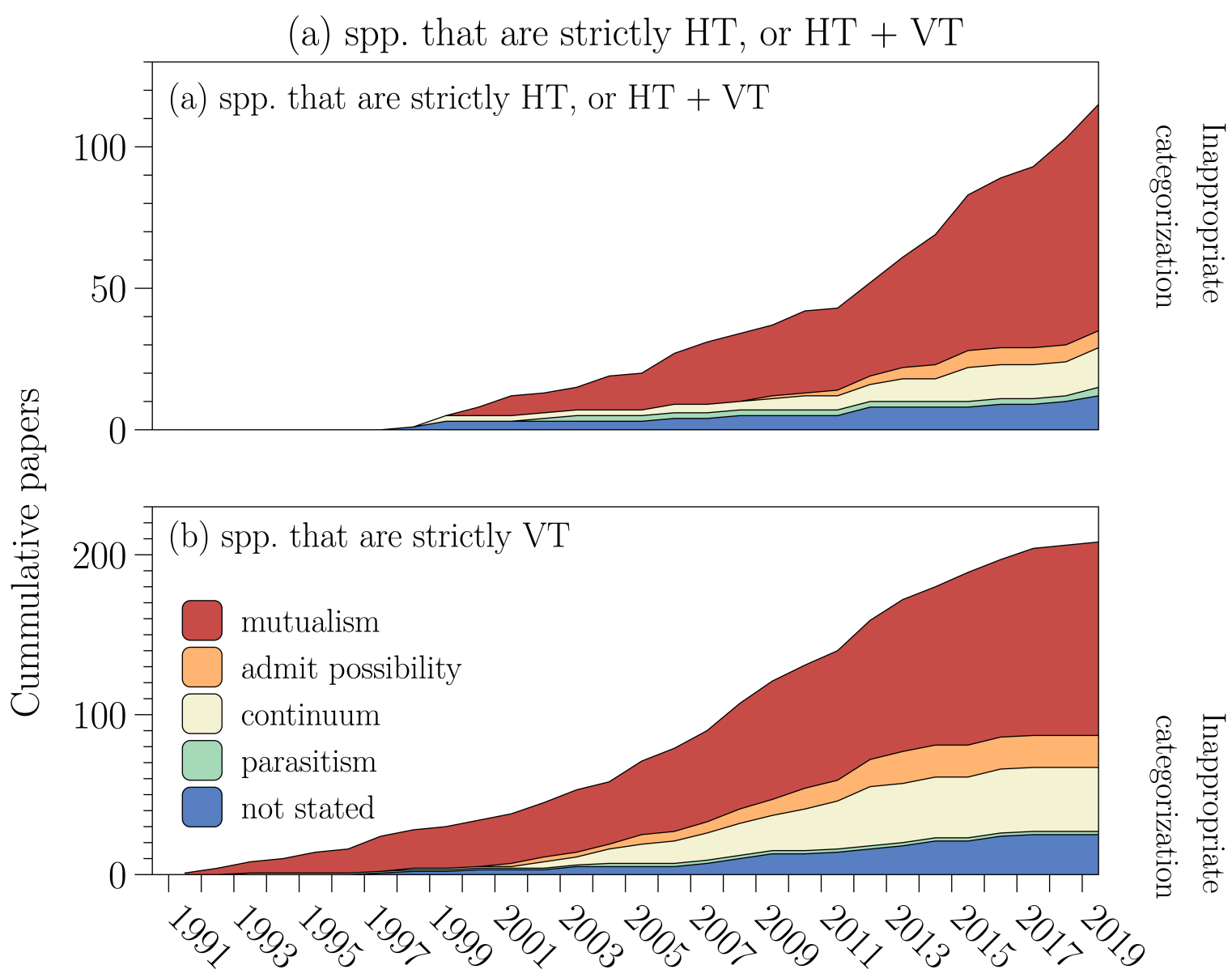

\section{Year}

Figure A.1: The cumulative number of research papers published on Epichloid species, cross classified by the transmission mode(s) of the subject species (according to Leuchtmann et al. 2014), and how the authors describe the relationship between the Epichloid species and the host grass plant. (a) denotes the cumulative number of papers that have as their subject matter an Epichloë species that is either strictly horizontally transmitted (HT) or has both horizontal and vertical transmission (HT $+\mathrm{VT}$ ); (b) denotes the same information where the focal species is strictly vertically transmitted (VT). Note that in (a) it is inappropriate to refer to these interactions as mutualisms (they could be, but equally they could be parasites), while in (b) it is inappropriate to refer to these interactions as anything other than a mutualism. 


\section{Appendix B Linear Stability of Fixed Point Solutions}

\section{Subsection B.1 General state equations}

$$
\begin{aligned}
& g_{i}(x, y)=\frac{\mathrm{d} x}{\mathrm{~d} t}, \\
& g_{j}(x, y)=\frac{\mathrm{d} y}{\mathrm{~d} t} .
\end{aligned}
$$

Note that the general equations are equations (6a) and (6b) in the main text, and Cases $\mathrm{A}$ and $\mathrm{B}$ can be retrieved by simply setting $\ell=1$ or $h=0$ respectively.

\section{Subsection B.2 Linear stability of fixed point solutions}

To evaluate the linear stability of the fixed point solutions, we substitute the fixed points into the relevant Jacobian matrix and then calculate the Eigenvalues. If the real parts are both negative then the fixed point is a stable equilibrium. The Jacobian is given by:

$$
\boldsymbol{J}=\left[\begin{array}{ll}
\frac{\partial g_{i}(x, y)}{\partial x} & \frac{\partial g_{i}(x, y)}{\partial y} \\
\frac{\partial g_{j}(x, y)}{\partial x} & \frac{\partial g_{j}(x, y)}{\partial y}
\end{array}\right]
$$

To calculate the Eigenvalues we need:

$$
\begin{aligned}
\boldsymbol{J} \vec{z} & =\lambda \vec{z}, \\
(\boldsymbol{J}-\lambda \boldsymbol{I}) \vec{z} & =\overrightarrow{0},
\end{aligned}
$$

where $\boldsymbol{I}$ is a $2 \times 2$ identity matrix and $\vec{z}$ is a vector of length 2 . The characteristic equation is then given by:

$$
\begin{aligned}
\operatorname{det}(\boldsymbol{J}-\lambda \boldsymbol{I}) & =0 \\
\operatorname{det}\left[\begin{array}{cc}
j_{11}-\lambda & j_{12} \\
j_{21} & j_{22}-\lambda
\end{array}\right] & =0 .
\end{aligned}
$$

From here we can obtain the characteristic polynomial:

$$
\lambda^{2}-\left(j_{11}+j_{22}\right) \lambda+\left(j_{11} j_{22}-j_{12} j_{21}\right)=0
$$

Eq. (23) always has two roots. These roots can be real or complex, and they do not have to be distinct. Employing the quadratic formula we can calculate the roots of Eq. (23) as:

$$
-\left(j_{11}+j_{22}\right) \pm \frac{\sqrt{\left(j_{11}+j_{22}\right)^{2}-4\left(j_{11} j_{22}-j_{12} j_{21}\right)}}{2}
$$

The fixed point solutions to the system of equations are stable if the real parts of the Eigenvalues are both negative. 
Subsection B.3 Case A. Strictly horizontal transmission, $\ell=1$

$$
\begin{aligned}
& g_{1}(x, y)=h x y-(x+y) c d x \\
& g_{2}(x, y)=(1-x-y)(a b x+b y)-h x y-(x+y) d y .
\end{aligned}
$$

Now calculate the Jacobian matrix. To do this we calculate the partial derivatives:

$$
\begin{aligned}
& \frac{\partial g_{1}(x, y)}{\partial x}=h y-c d(2 x+y), \\
& \frac{\partial g_{1}(x, y)}{\partial y}=x(h-c d), \\
& \frac{\partial g_{2}(x, y)}{\partial x}=y(-(b+d+h))-a b(2 x+y-1), \\
& \frac{\partial g_{2}(x, y)}{\partial y}=-b(a x+x+2 y-1)-d(x+2 y)-h x .
\end{aligned}
$$

Which yields:

$$
\boldsymbol{J}_{\boldsymbol{A}}=\left[\begin{array}{cc}
h y-c d(2 x+y) & x(h-c d) \\
y(-(b+d+h))-a b(2 x+y-1) & -b(a x+x+2 y-1)-d(x+2 y)-h x
\end{array}\right] .
$$

Next we calculate the Eigenvalues:

$$
\begin{aligned}
& \lambda_{A_{1}}=\frac{1}{2}\{-\sqrt{\mathfrak{e}-4(\mathfrak{f}+\mathfrak{g})}+\mathfrak{a}\}, \\
& \lambda_{A_{2}}=\frac{1}{2}\{\sqrt{\mathfrak{e}-4(\mathfrak{f}+\mathfrak{g})}+\mathfrak{a}\}
\end{aligned}
$$

where

$$
\begin{aligned}
\mathfrak{a}= & -a b x-b x-2 b y+b-2 c d x-c d y-d x-2 d y \\
& \quad-h x+h y \\
\mathfrak{e}= & (a b x+b x+2 b y-b+2 c d x+c d y+d x+2 d y \\
& \quad+h x-h y)^{2}, \\
\mathfrak{f}= & a b c d x+2 a b h x^{2}-a b h x+2 b c d x^{2}+4 b c d x y \\
& \quad-2 b c d x+2 b c d y^{2}, \\
\mathfrak{g}=- & b c d y-2 b h y^{2}+b h y+2 c d^{2} x^{2}+4 c d^{2} x y+2 c d^{2} y^{2} \\
& +2 c d h x^{2}-2 d h y^{2} .
\end{aligned}
$$

Using the solutions depicted in Fig. 4, it can be easily shown numerically that for these fixed point solutions the real parts of the two Eigenvalues are both negative, and therefore these solutions are stable.

Subsection B.4 Case B. Strictly vertically transmitted, $h=0$

$$
\begin{aligned}
& g_{3}(x, y)=(1-\ell)(1-x-y) a b x-(x+y) c d x \\
& g_{4}(x, y)=\ell(1-x-y) a b x+(1-x-y) b y-(x+y) d y
\end{aligned}
$$


The partial derivatives are then:

$$
\begin{aligned}
& \frac{\partial g_{3}(x, y)}{\partial x}=a b(\ell-1)(2 x+y-1)-c d(2 x+y) \\
& \frac{\partial g_{3}(x, y)}{\partial y}=x(a b(\ell-1)-c d) \\
& \frac{\partial g_{4}(x, y)}{\partial x}=-b(a \ell(2 x-1)+a \ell y+y)-d y \\
& \frac{\partial g_{4}(x, y)}{\partial y}=-b(a \ell x+x+2 y-1)-d(x+2 y) .
\end{aligned}
$$

Which yields:

$$
\boldsymbol{J}_{\boldsymbol{B}}=\left[\begin{array}{cc}
a b(\ell-1)(2 x+y-1)-c d(2 x+y) & x(a b(\ell-1)-c d) \\
-b(a \ell(2 x-1)+a \ell y+y)-d & -b(a \ell x+x+2 y-1)-d(x+2 y)
\end{array}\right] .
$$

The Eigenvalues are then:

$$
\begin{aligned}
& \lambda_{B_{1}}=\frac{1}{2}\{-\sqrt{\mathfrak{h}-4(\mathfrak{i}+\mathfrak{j})}+\mathfrak{k}\}, \\
& \lambda_{B_{2}}=\frac{1}{2}\{\sqrt{\mathfrak{h}-4(\mathfrak{i}+\mathfrak{j})}+\mathfrak{k}\},
\end{aligned}
$$

where

$$
\begin{aligned}
\mathfrak{h}=( & a b \ell-a b \ell x+2 a b x-a b \ell y+a b y-a b+b x+2 b y-b+2 c d x \\
& +c d y+d x+2 d y)^{2}, \\
\mathfrak{i}=- & a b^{2} \ell-2 a b^{2} \ell x^{2}+2 a b^{2} x^{2}+3 a b^{2} \ell x-4 a b^{2} \ell x y+4 a b^{2} x y \\
& -3 a b^{2} x-2 a b^{2} \ell y^{2}+2 a b^{2} y^{2}+3 a b^{2} \ell y-3 a b^{2} y \\
& +a b^{2}+a b c d \ell x \\
\mathfrak{j}=- & 2 a b d \ell x^{2}+2 a b d x^{2}+a b d \ell x-4 a b d \ell x y+4 a b d x y-a b d x \\
& -2 a b d \ell y^{2}+2 a b d y^{2}+2 a b d \ell y-2 a b d y+2 b c d x^{2} \\
& +4 b c d x y-2 b c d x+2 b c d y^{2}-b c d y+2 c d^{2} x^{2}+4 c d^{2} x y \\
& +2 c d^{2} y^{2}, \\
\mathfrak{k}=- & a b \ell+a b \ell x-2 a b x+a b \ell y-a b y+a b-b x-2 b y+b \\
& -2 c d x-c d y-d x-2 d y .
\end{aligned}
$$

Subsection B.5 Case C. Both horizontally and vertically transmitted, $0<\ell<1$ and $0<h$

$$
\begin{aligned}
& g_{5}(x, y)=(1-\ell)(1-x-y) a b x+h x y-(x+y) c d x \\
& g_{6}(x, y)=\ell(1-x-y)(a b x+b y)-h x y-(x+y) d y
\end{aligned}
$$

The partial derivatives are then:

$$
\begin{aligned}
& \frac{\partial g_{5}(x, y)}{\partial x}=a b(\ell-1)(2 x+y-1)-c d(2 x+y)+h y \\
& \frac{\partial g_{5}(x, y)}{\partial y}=x(a b(\ell-1)-c d+h) \\
& \frac{\partial g_{6}(x, y)}{\partial x}=a b(\ell-1)(2 x+y-1)-y(b+d+h), \\
& \frac{\partial g_{6}(x, y)}{\partial y}=b x(a(\ell-1)-1)-2 b y+b-d(x+2 y)-h x
\end{aligned}
$$


Which yields:

$$
\boldsymbol{J}_{\boldsymbol{C}}=\left[\begin{array}{cc}
a b(\ell-1)(2 x+y-1)-c d(2 x+y)+h y & x(a b(\ell-1)-c d+h) \\
a b(\ell-1)(2 x+y-1)-y(b+d+h) & b x(a(\ell-1)-1)-2 b y+b-d(x+2 y)-h x
\end{array}\right]
$$

The Eigenvalues are then:

$$
\begin{aligned}
& \lambda_{C_{1}}=\frac{1}{2}\{-\sqrt{\mathfrak{l}-4(\mathfrak{m}+\mathfrak{n}+\mathfrak{o}+\mathfrak{p})}+\mathfrak{q}\}, \\
& \lambda_{C_{2}}=\frac{1}{2}\{\sqrt{\mathfrak{l}-4(\mathfrak{m}+\mathfrak{n}+\mathfrak{o}+\mathfrak{p})}+\mathfrak{q}\},
\end{aligned}
$$

where

$$
\begin{aligned}
\mathfrak{l}=( & a b \ell-3 a b \ell x+3 a b x-a b \ell y+a b y-a b+b x+2 b y-b \\
& +2 c d x+c d y+d x+2 d y+h x-h y)^{2} \\
\mathfrak{m}=- & a b^{2} \ell-2 a b^{2} \ell x^{2}+2 a b^{2} x^{2}+3 a b^{2} \ell x-4 a b^{2} \ell x y+4 a b^{2} x y \\
& -3 a b^{2} x-2 a b^{2} \ell y^{2}+2 a b^{2} y^{2} \\
\mathfrak{n}=+ & 3 a b^{2} \ell y-3 a b^{2} y+a b^{2}-a b c d \ell x+a b c d x-2 a b d \ell x^{2} \\
& +2 a b d x^{2}+a b d \ell x-4 a b d \ell x y+4 a b d x y \\
\mathfrak{o}=- & a b d x-2 a b d \ell y^{2}+2 a b d y^{2}+2 a b d \ell y-2 a b d y-4 a b h \ell x^{2} \\
& +4 a b h x^{2}+2 a b h \ell x-2 a b h x+2 b c d x^{2}, \\
\mathfrak{p}=+ & 4 b c d x y-2 b c d x+2 b c d y^{2}-b c d y-2 b h y^{2}+b h y \\
& +2 c d^{2} x^{2}+4 c d^{2} x y+2 c d^{2} y^{2}+2 c d h x^{2}-2 d h y^{2}, \\
\mathfrak{q}=- & a b \ell+3 a b \ell x-3 a b x+a b \ell y-a b y+a b-b x-2 b y \\
& +b-2 c d x-c d y-d x-2 d y-h x+h y .
\end{aligned}
$$

Since we solved Eq. (9) numerically for Case C, we evaluated the stability of these solutions by substituting the numerical solutions into Eq. (37) and checking the signs of the real parts of the Eigenvalues. All feasible solutions were stable. 


\section{Appendix C Solution B.ii.2 Conditions}

In this appendix, we show that the conditions on the coexistence solution (Eq. (15)) never constrained this solution, given biologically feasible parameter values. Equation 17 requires that:

$$
(\mathrm{c} 1): c(b+d) \neq 0 \text {. }
$$

Since $0<c, 0<b$, and $0<d, c(b+d)$ can never equal 0 ; so this condition is always met. Equation 17 also requires that:

$$
(\mathrm{c} 2): b d(a(c-1) \ell+a-c)(a b(\ell-1)-c d) \neq 0 .
$$

It can be shown that (c2) is met if the following are true:

$$
\begin{gathered}
(\mathrm{c} 2 . \mathrm{i}): b d \neq 0, \\
(\mathrm{c} 2 . \mathrm{ii}): c \neq \frac{a b(\ell-1)}{d}, \\
(\mathrm{c} 2 . \mathrm{iii}): c \neq \frac{a \ell-a}{a \ell-1} .
\end{gathered}
$$

Since $0<b$ and $0<d$, (c2.i) is always true. Since $\ell<1$, condition (c2.ii) would require $c<0$ for it to equal the right hand side of the equation and so, since biologically $0<c$, (c2.ii) is always true. Condition (c2.iii) can be false in two further sets of conditions:

$$
\begin{aligned}
& \text { (c2.iii.a) : } a=c=1, \quad \ell<1, \text { or } \\
& \text { (c2.iii.b) : } a c \neq a, \quad \ell=\frac{a-c}{a(1-c)} .
\end{aligned}
$$

Condition (c2.iii.a) denotes the biologically unlikely case in which the endophyte imposes no advantages or disadvantages at all. This case is unlikely since stochastic processes (akin to genetic drift in a population genetics model) would ensure that, eventually, the endophyte would be lost from the system. Next, we will show that the conditions that support a coexistence solution are such that (c2.iii.b) never constrains the solution space, and hence for coexistence, the solution to Eq. (17) can be true subject to the constraints $0<\ell<(a-c) / a$ and $c<a$.

Using the Eq. (1) for $\phi^{*}$, a coexistence solution requires that:

$$
0<\phi^{*}=\frac{-a b(\ell-1)(a(\ell-1)+c)}{-a b(\ell-1)(a(\ell-1)+c)+a^{2} b c(\ell-1) \ell}<1 .
$$

Equation 45 can be simplified to:

$$
0<\phi^{*}=-\frac{a \ell-a+c}{a c \ell-a \ell+a-c}<1 .
$$

Equation 46 is true iff both of the following conditions hold:

$$
0<c<a, \quad 0<\ell<\frac{a-c}{a}
$$




\section{Acknowledgments}

Wilfrid Laurier University and its campuses are located on the Haldimand tract, traditional territory of the Neutral, Anishnaabe and Haudenosaunee peoples. We recognize, honour, and respect these nations as the traditional stewards of the lands and water on which Laurier is now present. This research was supported by grants to JAN from the Canadian Natural Science and Engineering Research Council (NSERC) and the Ontario Ministry of Agriculture, Food, and Rural Affairs (OMAFRA). We thank several anonymous referees for helpful comments on an earlier version of this manuscript.

\section{Literature cited}

Afkhami, Michelle E., and Jennifer A. Rudgers. 2008. "Symbiosis Lost: Imperfect Vertical Transmission of Fungal Endophytes in Grasses." The American Naturalist 172 (3): 405-416. https://doi. org/10.1086/589893.

Anderson, Roy M., and Robert M. May. 1992. Infectious Diseases of Humans: Dynamics and Control. Oxford University Press.

Antunes, P. M., J. Miller, L. M. Carvalho, J. N. Klironomos, and J. A. Newman. 2008. "Even After Death the Endophytic Fungus of Schedonorus phoenix Reduces the Arbuscular Mycorrhizas of Other Plants." Functional Ecology 22 (5): 912-918. https://doi.org/10.1111/j.1365-2435.2008. 01432.x.

Aris, Rutherford. 1994. Mathematical Modelling Techniques. Dover Press.

Bastías, Daniel A., M. Alejandra Martínez-Ghersa, Jonathan A. Newman, Stuart D. Card, Wade J. Mace, and Pedro E. Gundel. 2018. "Jasmonic Acid Regulation of the Anti-Herbivory Mechanism Conferred by Fungal Endophytes in Grasses.” Journal of Ecology 106 (6): 2365-2379. https: //doi.org/10.1111/1365-2745.12990.

Black, Andrew J., and Alan J. Mckane. 2012. "Stochastic Formulation of Ecological Models and Their Applications." Trends in Ecology and Evolution 27 (6): 337-345. https://doi.org/10.1016/j.tree. 2012.01.014.

Bullock, J. M., B. Clear Hill, and J. Silvertown. 1994. "Tiller Dynamics of Two Grasses-Responses to Grazing, Density and Weather." Journal of Ecology 82:331-340. https://doi.org/10.2307/ 2261301.

Carrol, G. C. 1988. "Fungal Endophytes in Stems and Leaves: From Latent Pathogen to Mutualistic Symbiont.” Ecology 69:2-9. https://doi.org/10.2307/1943154.

Cheplick, G. P., A. Perera, and K. Koulouris. 2000. "Effect of Drought on the Growth of Lolium perenne Genotypes with and Without Fungal Endophytes.” Functional Ecology 14 (6): 657-667. https://doi.org/10.1046/j.1365-2435.2000.00466.x.

Cheplick, Gregory P. 2007. "Costs of Fungal Endophyte Infection in Lolium perenne Genotypes From Eurasia and North Africa Under Extreme Resource Limitation." Environmental and Experimental Botany 60 (2): 202-210. https://doi.org/10.1016/j.envexpbot.2006.10.001.

Cheplick, Gregory P. 2008. "Host Genotype Overrides Fungal Endophyte Infection in Influencing Tiller and Spike Production of Lolium perenne (Poaceae) in a Common Garden Experiment.” American Journal of Botany 95 (9): 1063-1071. https://doi.org/10.3732/ajb.0800042.

Christensen, Michael J., Raymond J. Bennett, and Jan Schmid. 2002. "Growth of Epichloë/Neotyphodium and P-Endophytes in Leaves of Lolium and Festuca Grasses." Mycological Research 106 (1): 93-106. https://doi.org/10.1017/s095375620100510x. 
Clay, Keith. 1988. "Fungal Endophytes of Grasses: A Defensive Mutualism Between Plants and Fungi.” Ecology 69 (1): 10-16. https://doi.org/10.2307/1943155.

Clay, Keith. 1991. "Endophytes as Antagonists of Plant Pests." In Microbial Ecology of Leaves, edited by J. H. Andrews and S. S. Hirano, 331-57. New York: Springer.

Clay, Keith. 1993. "The Ecology and Evolution of Endophytes." Agriculture, Ecosystems and Environment 44 (1-4): 39-64. https://doi.org/10.1016/0167-8809(93)90038-q.

Clay, Keith, Tad N. Hardy, and Abner M. Hammond. 1985. "Fungal Endophytes of Grasses and Their Effects on an Insect Herbivore.” Oecologia 66 (1): 1-5. https://doi.org/10.1007/bf00378545.

Clay, Keith, and Christopher L. Schard1. 2002. "Evolutionary Origins and Ecological Consequences of Endophyte Symbiosis with Grasses." The American Naturalist 160 (S4): S99-S127. https://doi. org/10.1086/342161.

Darbyshire, Stephen J., and Leon E. Pavlickf. 2020. "Festuca arizonica." In Flora of North America. July. http://dev.floranorthamerica.org/Festuca_arizonica.

Dawkins, Richard. 1978. The Selfish Gene. Granada Publishing Company.

Ewald, Paul W. 1987. "Transmission Modes and Evolution of the Parasitism-Mutualism Continuum.” Annals of the Nerw York Academy of Sciences 503 (1): 295-306. https://doi.org/10.1111/j.17496632.1987.tb40616.x.

Faeth, Stanley H. 2002. “Are Endophytic Fungi Defensive Plant Mutualists?” Oikos 98 (1): 25-36. https://doi.org/10.1034/j.1600-0706.2002.980103.x.

Faeth, Stanley H. 2010. “Occam's Razor Cuts Both Ways: Endophytes, Resource Allocation, Herbivory, and Mutualism: A Reply to Rudgers et al.” The American Naturalist 176 (1): 104-110. https://doi.org/10.1086/652997.

Faeth, Stanley H., and William F. Fagan. 2002. "Fungal Endophytes: Common Host Plant Symbionts but Uncommon Mutualists." Integrative and Comparative Biology 42 (2): 360-368. https://doi. org/10.1093/icb/42.2.360.

Faeth, Stanley H., Karl P. Hadeler, and Horst R. Thieme. 2007. "An Apparent Paradox of Horizontal and Vertical Disease Transmission.” Journal of Biological Dynamics 1 (1): 45-62. https://doi.org/ 10.1080/17513750601040367.

Faeth, Stanley H., and Cyd E. Hamilton. 2006. "Does an Asexual Endophyte Symbiont Alter Life Stage and Long-Term Survival in a Perennial Host Grass?" Microbial Ecology 52 (4): 748-755. https://doi.org/10.1007/s00248-006-9123-z.

Faeth, Stanley H., Marjo L. Helander, and Kari T. Saikkonen. 2004. "Asexual Neotyphodium Endophytes in a Native Grass Reduce Competitive Abilities.” Ecology Letters 7 (4): 304-313. https: 7/doi.org/10.1111/j.1461-0248.2004.00578.x.

Faeth, Stanley H., and T. J. Sullivan. 2003. "Mutualistic Asexual Endophytes in a Native Grass Are Usually Parasitic.” The American Naturalist 161 (2): 310-325. https://doi.org/10.1086/345937.

Faeth, Stanley H., T. J. Sullivan, and C. E. Hamilton. 2000. "What Maintains High Levels of Neotyphodium Endophytes in Native Grasses? A Dissenting View and Alternative Hypotheses." In Proceedings of the 4th International Neotyphodium/Grass Interactions Symposium, Soest, Germany, 2729.

Faeth, Stanley H., D. Wilson, M. Helander, K. Saikkonen, F. Schulthess, and T. J. Sullivan. 1997. "Neotyphodium in Native Populations of Arizona Fescue: A Nonmutualis?" In Neotyphodium/Grass Interactions, edited by Charles W. Bacon and Nicholas S. Hill, 165-66. New York: Plenum Press.

๑ OPEN ACCESS - PTPBIO.ORG 
Fine, Paul E. M. 1975. "Vectors and Vertical Transmission: An Epidemiologic Perspective." Annals of the New York Academy of Sciences 266 (1): 173-194. https://doi.org/10.1111/j.1749-6632.1975. tb35099.x.

Fuchs, Benjamin, Markus Krischke, Martin J. Mueller, and Jochen Krauss. 2013. "Peramine and Lolitrem B. From Endophyte-Grass Associations Cascade Up the Food Chain.” Journal of Chemical Ecology 39 (11-12): 1385-1389. https://doi.org/10.1007/s10886-013-0364-2.

Geddes-McAlister, Jennifer, Arjun Sukumaran, Aurora Patchett, Heather A. Hager, Jenna Dale, Jennifer L. Roloson, Nicholas Prudhomme, et al. 2020. "Examining the Impacts of $\mathrm{CO}_{2}$ Concentration and Genetic Compatibility on Perennial Ryegrass-Epichloë festucae var. lolii Interactions." Journal of Fungi 6 (4): 360. https://doi.org/10.3390/jof6040360.

Gundel, Pedro E., William B. Batista, Marcos Texeira, M. Alejandra Martínez-Ghersa, Marina Omacini, and Claudio M. Ghersa. 2008. "Neotyphodium Endophyte Infection Frequency in Annual Grass Populations: Relative Importance of Mutualism and Transmission Efficiency.” Proceedings of the Royal Society of London B. Biological Sciences 275 (1637): 897-905. https://doi.org/ 10.1098/rspb.2007.1494.

Härri, Simone A., Jochen Krauss, and Christine B. Müller. 2008a. "Fungal Endosymbionts of Plants Reduce Lifespan of an Aphid Secondary Parasitoid and Influence Host Selection.” Proceedings of the Royal Society B. Biological Sciences 275 (1651): 2627-2632. https://doi.org/10.1098/rspb.2008. 0594 .

Härri, Simone A., Jochen Krauss, and Christine B. Müller. 2008b. "Trophic Cascades Initiated by Fungal Plant Endosymbionts Impair Reproductive Performance of Parasitoids in the Second Generation." Oecologia 157 (3): 399-407. https://doi.org/10.1007/s00442-008-1094-y.

Hosseini, F., M. R. Mosaddeghi, M. A. Hajabbasi, and M. A. Sabzalian. 2016. "Role of Fungal Endophyte of Tall Fescue (Epichloë coenophiala) on Water Availability, Wilting Point and Integral Energy in Texturally-Different Soils." Agricultural Water Management 163:197-211. https://doi. org/10.1016/j.agwat.2015.09.024

Hunt, Matthew G., and Jonathan A. Newman. 2005. "Reduced Herbivore Resistance From a Novel Grass-Endophyte Association.” Journal of Applied Ecology 42 (4): 762-769. https://doi.org/10. 1111/j.1365-2664.2005.01061.x.

Johnson, Nancy C., J-H Graham, and F. A. Smith. 1997. "Functioning of Mycorrhizal Associations Along the Mutualism-Parasitism Continuum.” Nerw Phytologist 135 (4): 575-585. https://doi. org/10.1046/j.1469-8137.1997.00729.x.

Leuchtmann, Adrian, Charles W. Bacon, Christopher L. Schardl, James F. White Jr, and Mariusz Tadych. 2014. "Nomenclatural Realignment of Neotyphodium Species with Genus Epichloë." Mycologia 106 (2): 202-215. https://doi.org/10.3852/13-251.

Levins, Richard. 1966. "The Strategy of Model Building in Population Biology." American Scientist 54 (4): 421-431.

Lewis, G. C., C. Ravel, W. Naffaa, C. Astier, and G. Charmet. 1997. "Occurrence of Acremonium Endophytes in Wild Populations of Lolium spp. in European Countries and a Relationship Between Level of Infection and Climate in France." Annals of Applied Biology 130 (2): 227-238. https://doi.org/10.1111/j.1744-7348.1997.tb06828.x.

Lopez, Jorge E., Stanley H. Faeth, and Myrna Miller. 1995. "Effect of Endophytic Fungi on Herbivory by Redlegged Grasshoppers (Orthoptera: Acrididae) on Arizona Fescue." Environmental Entomology 24 (6): 1576-1580. https://doi.org/10.1093/ee/24.6.1576. 
Müller, Christine B., and Jochen Krauss. 2005. "Symbiosis Between Grasses and Asexual Fungal Endophytes.” Current Opinion in Plant Biology 8 (4): 450-456. https://doi.org/10.1016/j.pbi.2005. 05.007.

Newman, J. A., M. L. Abner, R. G. Dado, D. J. Gibson, A. Brookings, and A. J. Parsons. 2003. "Effects of Elevated $\mathrm{CO}_{2}$, Nitrogen and Fungal Endophyte-Infection on Tall Fescue: Growth, Photosynthesis, Chemical Composition and Digestibility." Global Change Biology 9 (3): 425-437. https://doi.org/10.1046/j.1365-2486.2003.00601.x.

Newman, Jonathan A. 1991. "Patch Use Under Predation Hazard: Foraging Behavior in a Simple Stochastic Environment.” Oikos 61 (1): 29-44. https://doi.org/10.2307/3545404.

Olejniczak, Paweł, and Marlena Lembicz. 2007. "Age-Specific Response of the Grass Puccinellia distans to the Presence of a Fungal Endophyte.” Oecologia 152 (3): 485-494. https://doi.org/10. 1007/s00442-007-0660-z.

Patchett, Aurora, and Jonathan A. Newman. 2021. "Comparison of Plant Metabolites in Root Exudates of Lolium perenne Infected with Different Strains of the Fungal Endophyte Epichloë festucae var. lolii." Journal of Fungi 7 (2): 148. https://doi.org/10.3390/jof7020148.

Rabinowitz, Deborah, and Jody K. Rapp. 1981. "Dispersal Abilities of Seven Sparse and Common Grasses From a Missouri Prairie.” American Journal of Botany 68 (5): 616-624. https://doi.org/10. 1002/j.1537-2197.1981.tb12393.x.

Raman, Anantanarayanan, and Trichur Subramanian Suryanarayanan. 2017. "Fungus-Plant Interaction Influences Plant-Feeding Insects." Fungal Ecology 29:123-132. https://doi.org/10.1016/j. funeco.2017.06.004.

Rasmussen, Susanne, Anthony J. Parsons, Shalome Bassett, Michael J. Christensen, David E. Hume, Linda J. Johnson, Richard D. Johnson, et al. 2007. "High Nitrogen Supply and Carbohydrate Content Reduce Fungal Endophyte and Alkaloid Concentration in Lolium perenne." Nerw Phytologist 173 (4): 787-797. https://doi.org/10.1111/j.1469-8137.2006.01960.x.

Rasmussen, Susanne, Anthony J. Parsons, Karl Fraser, Hong Xue, and Jonathan A. Newman. 2008. "Metabolic Profiles of Lolium perenne Are Differentially Affected by Nitrogen Supply, Carbohydrate Content, and Fungal Endophyte Infection." Plant Physiology 146 (3): 1440-1453. https: //doi.org/10.1104/pp.107.111898.

Rasmussen, Susanne, Anthony J. Parsons, Alison Popay, Hong Xue, and Jonathan A. Newman. 2008. "Plant-Endophyte-Herbivore Interactions: More Than Just Alkaloids?" Plant Signaling and Behavior 3 (11): 974-977. https://doi.org/10.4161/psb.6171.

Ravel, Catherine, Yannis Michalakis, and Gilles Charmet. 1997. "The Effect of Imperfect Transmission on the Frequency of Mutualistic Seed-Borne Endophytes in Natural Populations of Grasses." Oikos 80 (1): 18-24. https://doi.org/10.2307/3546511.

Rudgers, Jennifer A., Andrew J. Davitt, Keith Clay, Pedro E. Gundel, and Marina Omacini. 2010. "Searching for Evidence Against the Mutualistic Nature of Hereditary Symbioses: A Comment on Faeth.” The American Naturalist 176 (1): 99-103. https://doi.org/10.1086/652996.

Rudgers, Jennifer A., Tom E. X. Miller, Shaun M. Ziegler, and Kelly D. Craven. 2012. "There Are Many Ways to Be a Mutualist: Endophytic Fungus Reduces Plant Survival but Increases Population Growth." Ecology 93 (3): 565-574. https://doi.org/10.1890/11-0689.1. 
Ryan, Geraldine D., Kruti Shukla, Susanne Rasmussen, Barry J. Shelp, and Jonathan A. Newman. 2014. "Phloem Phytochemistry and Aphid Responses to Elevated $\mathrm{CO}_{2}$, Nitrogen Fertilization and Endophyte Infection." Agricultural and Forest Entomology 16 (3): 273-283. https://doi.org/10. 1111/afe.12055.

Saikkonen, Kari, Stanley H. Faeth, M. Helander, and T. J. Sullivan. 1998. "Fungal Endophytes: A Continuum of Interactions with Host Plants." Annual Review of Ecology and Systematics 29 (1): 319-343. https://doi.org/10.1146/annurev.ecolsys.29.1.319.

Saikkonen, Kari, M. Helander, Stanley H. Faeth, F. Schulthess, and D. Wilson. 1999. "EndophyteGrass-Herbivore Interactions: The Case of Neotyphodium Endophytes in Arizona Fescue Populations." Oecologia 121 (3): 411-420. https://doi.org/10.1007/s004420050946.

Saikkonen, Kari, Diana Ion, and Mats Gyllenberg. 2002. "The Persistence of Vertically Transmitted Fungi in Grass Metapopulations." Proceedings of the Royal Society of London B. Biological Sciences 269 (1498): 1397-1403. https://doi.org/10.1098/rspb.2002.2006.

Saikkonen, Kari, Piippa Wäli, Marjo Helander, and Stanley H. Faeth. 2004. "Evolution of Endophyte-Plant Symbioses.” Trends in Plant Science 9 (6): 275-280. https://doi.org/10.1016/j. tplants.2004.04.005.

Schardl, Christopher L. 2010. “The Epichloae, Symbionts of the Grass Subfamily Poöideae.” Annals of the Missouri Botanical Garden 97 (4): 646-665. https://doi.org/10.3417/2009144.

Schardl, Christopher L., Kelly D. Craven, S. Speakman, Arnold Stromberg, A. Lindstrom, and Ruriko Yoshida. 2008. "A Novel Test for Host-Symbiont Codivergence Indicates Ancient Origin of Fungal Endophytes in Grasses.” Systematic Biology 57 (3): 483-498. https://doi.org/10. $1080 / 10635150802172184$.

Schardl, Christopher L., Simona Florea, Juan Pan, Padmaja Nagabhyru, Sladana Bec, and Patrick J. Calie. 2013. "The Epichloae: Alkaloid Diversity and Roles in Symbiosis with Grasses." Current Opinion in Plant Biology 16 (4): 480-488. https://doi.org/10.1016/j.pbi.2013.06.012.

Shukla, Kruti, Heather A. Hager, Kathryn A. Yurkonis, and Jonathan A. Newman. 2015. "Effects of the Epichloë Fungal Endophyte Symbiosis with Schedonorus pratensis on Host Grass Invasiveness." Ecology and Evolution 5 (13): 2596-2607. https://doi.org/10.1002/ece3.1536.

Smith, F. Andrew, and Sally E. Smith. 2013. "How Useful Is the Mutualism-Parasitism Continuum of Arbuscular Mycorrhizal Functioning?” Plant and Soil 363 (1-2): 7-18. https://doi.org/10.1007/ s11104-012-1583-y.

Soto-Barajas, Milton C., Iñigo Zabalgogeazcoa, Javier Gómez-Fuertes, Virginia González-Blanco, and Beatriz R. Vázquez-De-Aldana. 2016. "Epichloë Endophytes Affect the Nutrient and Fiber Content of Lolium perenne Regardless of Plant Genotype.” Plant and Soil 405 (1): 265-277. https ://doi.org/10.1007/s11104-015-2617-z.

Strong, D. R. 1988. “Endophytic Mutualism and Plant Protection From Herbivores.” Ecology 69:1. https://doi.org/10.2307/1943153.

Sullivan, T. J., and Stanley H. Faeth. 1999. "Neotyphodium in Arizona Fescue: A Necessary Symbiont?" American Zoologist 39 (5): 120A-120A. https://doi.org/10.1007/978-1-4899-0271-9_27.

Turelli, Michael. 1994. "Evolution of Incompatibility-Inducing Microbes and Their Hosts." Evolution 48 (5): 1500-1513. https://doi.org/10.2307/2410244.

White, Richard H., Milton C. Engelke, Sharon J. Morton, Jennifer M. Johnson-Cicalese, and Bridget A. Ruemmele. 1992. "Acremonium Endophyte Effects on Tall Fescue Drought Tolerance." Crop Science 32 (6): 1392-1396. https://doi.org/10.2135/cropsci1992.0011183x003200060017x. 
Zug, Roman, and Peter Hammerstein. 2015. "Bad Guys Turned Nice? A. Critical Assessment of Wolbachia Mutualisms in Arthropod Hosts.” Biological Reviews 90 (1): 89-111. https://doi.org/10. 1111/brv.12098.

(C) 2022 Author(s)

This is an open-access article distributed under the terms of the Creative Commons Attribution 4.0 International license, which permits anyone to download, copy, distribute, display, or adapt the text without asking for permission, provided that the creator(s) are given full credit.

ISSN 2475-3025 